\title{
Newsvendor Models for Innovative Products with One-Shot
}

\section{Decision Theory}

\author{
Peijun Guo*, Xiuyan Ma \\ Faculty of Business Administration, Yokohama National University \\ 79-4 Tokiwadai, Hodogaya-ku, Yokohama, 240-8501 Japan \\ guo@ynu.ac.jp,maxiuyan@gmail.com
}

\begin{abstract}
In this paper, newsvendor problems for innovative products are analyzed. Because the product is new, no relevant historical data is available for statistical demand analysis. Instead of using the probability distribution, the possibility distribution is utilized to characterize the uncertainty of the demand. We consider products whose life cycles are expected to be smaller than the procurement lead times. Determining optimal order quantities of such products is a typical one-shot decision problem for a retailer. Therefore, newsvendor models for innovative products are proposed based on the one-shot decision theory (OSDT). The main contributions of this research are as follows: the general solutions of active, passive, apprehensive and daring focus points and optimal alternatives are proposed and the existence theorem is established in the one-shot decision theory; a simple and effective approach for identifying the possibility distribution is developed; newsvendor models with four types of focus points are built; managerial insights into the behaviors of different types of retailers are gained by the theoretical analysis; the proposed models are scenario-based decision models which provide a fundamental alternative to analyze newsvendor problems for innovative products.
\end{abstract}

Keywords: Decision making, one-shot decision theory, newsvendor model, scenario-based decision model, focus point, innovative product

\section{Introduction}

The newsvendor problem is a well-known inventory management problem. It has the following characteristics. Prior to the season, the seller must decide the quantity of the goods to purchase/produce. The procurement lead-time tends to be quite long relative to the selling season. As a result, there's not enough opportunity to replenish inventory once the season has begun. Excess stock can only be salvaged at a loss once the season is over. The classical newsvendor problem is characterized by the fixed selling and wholesale prices and the uncertain demand of goods with a short life cycle, such as perishable items and fashion items. Its optimal 
order quantity is solved by the critical fractile of the demand distribution. A considerable amount of research (Grubbstrom, 2010; Wang, 2010; Caliskan-Demirag et al., 2011; Chen, 2011; Salinger and Ampudia, 2011; Xu et. al., 2011; Brito and de Almeida, 2012; Seifert et al., 2012; Summerfield and Dror, 2012; Murray et al. , 2012; Wang et al., 2012; Wu et al., 2012; Kwon and Cheong, 2014) and bibliographies have appeared in the newsvendor literature, including those of Petruzzi and Dada (1999), Khouja (1999) and Qin et al. (2011). Many extensions of the classic newsvendor problem, such as different demand functions, different supplier pricing policies to coordinate the supply chain, different retailer risk profits, supplier capacity constraints and multi-product cases have been made. But almost all the extensions have been made in the probabilistic framework where the uncertainty of the demand and the supply is characterized by probability distributions, and the objective function is used to maximize the expected utility or the probability measure of achieving a target profit.

Several researchers have analyzed newsvendor problems under a fuzzy environment. Petrovic et al. (1996) gave a fuzzy newsboy model where the overage cost, the shortage cost, and the demand were fuzzy numbers; and the optimal order quantity was obtained by the defuzzification of total costs. Ishii and Konno (1998) investigated the fuzzy newsboy problem in which the shortage cost was vague and expressed as a fuzzy number and an optimal order quantity was obtained by fuzzy maximal order. Kao and Hsu (2002) obtained the optimal order quantity to minimize the fuzzy cost by comparing the area of fuzzy numbers. Li et al. (2002) proposed two models, in one the demand was probabilistic while the costs were fuzzy and in the other the costs were deterministic but the demand was fuzzy; and the profit was maximized through ordering fuzzy numbers with respect to their total integral values. Xu and Zhai (2008) developed a newsvendor model with the fuzzy demand to examine the total profits of one-supplier and one-retailer supply chain in incorporative and cooperative situations. Dutta and Chakraborty (2010) modeled a two-item newsvendor problem with one-way substitution where the demand was a fuzzy number and the optimal order quantity was obtained by the interval-valued expectation method. Ryu and Yucesan (2010) built fuzzy newsvendor models to study three coordination policies: quantity discounts, profit sharing and buyback. Chen and Ho (2011) considered the incremental quantity discounts with the fuzzy demand and the optimal order quantity was determined by ranking fuzzy total costs.

We examine the newsvendor problem for the innovative product as defined by Fisher. According to Fisher (1997), products basically belong to either primarily functional category or innovative one. Functional products satisfy basic needs and have stable, predictable demand and long life cycles whereas innovative products have higher profit margins, intrinsically unpredictable demands and short life cycles. In addition, for such an innovative product, the procurement lead-time is usually longer than the selling season so that there is usually only one 
opportunity to order goods before the season. For example, Sport Obermeyer, a major supplier of fashion skiwear, ships its products in September, but has to commit itself to products well before February (Fisher, 1997). However, the retailer season is only a few months long. Hence, the newsvendor problems for innovative products can be regarded as one-shot decision problems, which are typical for situations where a decision is made only once under uncertainty.

Guo (2011) initially proposed the one-shot decision theory (OSDT) for one-shot decision problems. The one-shot decision theory provides a scenario-based choice instead of the lottery-based choices in the existing decision theories. As the applications, a duopoly market of a new product with a short life cycle and the private real estate investment were analyzed (Guo, 2010a; Guo, 2010b; Guo et al., 2010). Recently, the research (Guo, 2014) clarified the fundamental differences between the one-shot decision theory and other decision theories under uncertainty and pointed out the instinct problems in other decision theories to show that the one-shot decision theory is necessary to solve one-shot decision problems and manifested the relationship between the one-shot decision theory and the probabilistic decision methods. Guo and $\mathrm{Li}$ (2014) proposed multistage one-shot decision making approaches and analyzed the optimal stopping problem.

Because the product is innovative, no relevant historical data is available to obtain the probability distribution of the demand. We use the possibility distribution to characterize the uncertainty of the demand which reflects experts' judgment. Since the life cycle of the innovative product is shorter than the procurement lead-time, determining the optimal order quantity is a typical one-shot decision problem for the retailer. In this research, the one-shot decision theory (OSDT) based newsvendor models are proposed. In the proposed models, for each order quantity, the retailer chooses one demand amongst all possible demands while considering the satisfaction level caused by the occurrence of the demand and the possibility degree of the demand occurring. The selected demand is called the focus point of the order quantity. The optimal order quantity corresponds to the maximum satisfaction level of its focus point. We take into account four types of decision makers, i.e. active, passive, apprehensive and daring retailers who focus on the demand with a higher satisfaction and a higher possibility, the demand with a lower satisfaction and a higher possibility, the demand with a lower satisfaction and a lower possibility, the demand with a higher satisfaction and a lower possibility, respectively. The optimal order quantities for these four types of retailers are obtained and the theoretical analysis is made.

The contributions of this research are as follows: Obtaining the possibility distribution always poses a fundamental problem for decision making without enough historical data. We propose an optimization method with the pairwise comparison to identify the possibility distribution to reflect the judgment of the expert. With an assumption that the possibility distribution and the 
satisfaction function are quasi-concave, the general solutions of the focus points and the optimal alternatives are obtained and the existence theorem is established in the one-shot decision theory. The probabilistic newsvendor models seek the optimal order quantities to maximize the expected values or the probability measures. The fuzzy newsvendor models use the optimal order quantities to maximize the fuzzy profits. These two kinds of models take into account all demand values when determining the optimal order quantities. However, there is one and only one demand that will appear when the selling season comes. We build the newsvendor models with the one-shot decision theory which fit the one-shot feature of the retailer's order decision. The managerial insights into the behaviors of different types of retailers are gained by the theoretical analysis. The proposed methods provide a fundamental alternative to analyze the newsvendor problems for innovative products.

The remainder of the paper is organized as follows: In Section 2, the general solutions of the focus points and the optimal alternatives in the one-shot decision theory are proposed. In Section 3, newsvendor models for innovative products are developed based on the one-shot decision theory. In Section 4, the method for identifying the possibility distribution of the demand of an innovative product is proposed. In Section 5, the results of analysis of the proposed newsvendor models are given. In Section 6, a numerical example is used to demonstrate the proposed approach. Finally, the research conclusions are given in Section 7.

\section{General solutions in the one-shot decision theory (OSDT)}

According to the one-shot decision theory, a person makes a one-shot decision based on some particular scenario (state) which is the most appropriate one for him/her while considering the satisfaction level incurred by this scenario and its possibility degree. The one-shot decision process is separated into two steps. The first step is to seek an appropriate scenario from all possible states for each alternative. This scenario is called as the focus point of the alternative. The second step is to evaluate the alternatives by the satisfaction levels incurred by the focus points for obtaining the optimal alternative. Twelve kinds of focus points are proposed to characterize the different preferences of decision makers for selecting scenarios (Guo, 2011). In this paper, we consider only four types of focus points, that is, active, passive, apprehensive and daring focus points.

\subsection{Four types of focus points}

The set of a state $x$ is $S$. The degree to which a state $x$ is to occur in the future is characterized by a possibility distribution $\pi(x)$, as defined below.

Definition 1. Given a function $\pi: S \rightarrow[0,1]$, if $\max _{x \in S} \pi(x)=1$, then $\pi(x)$ is called a possibility distribution. 
The easy way to distinguish possibility and probability is that there is no additivity requirement for possibility. $\pi(x)=1$ means that it is normal that $x$ occurs but does not mean that $x$ is certain. $\pi(x)=0$ means that it is abnormal that $x$ occurs but does not mean it absolutely does not appear. The possibility distribution is a less restricted framework than single probability measures and hence can be used for encoding poorly known subjective probability information. Moreover, the possibility distribution might be effective for representing the rough knowledge or judgment of human being when the information is not rich enough. The methods for identifying possibility distributions based on similarity, potential surprise and voting were proposed in the literature (Guo and Tanaka, 2003; Guo et al., 2010; Guo, 2011).

The set of an alternative $a$ is $A$. The consequence resulting from the combination of an alternative $a$ and a state $x$ is referred to as a payoff, denoted as $v(x, a)$. The set of a payoff is $V$. The satisfaction level of a decision maker for a payoff is expressed by a satisfaction function, as defined below.

Definition 2. The function $u: V \rightarrow[0,1]$ is called a satisfaction function if it satisfies $u\left(v_{1}\right)>u\left(v_{2}\right)$ for $v_{1}>v_{2}$.

Because the payoff is a function of $x$ and $a$, we write the satisfaction function as $u(x, a)$. Since one and only one state will come up for a one-shot decision problem, a decision maker should decide which state ought to be considered for making a one-shot decision. How to determine focus points (focused states) depends on his/her attitudes about possibility and satisfaction. For characterizing the focus points, we introduce the following operators.

Definition 3. Given a vector $\left[b_{1}, b_{2}, \cdots, b_{n}\right], \min \left[b_{1}, b_{2}, \cdots, b_{n}\right]$ and $\max \left[b_{1}, b_{2}, \cdots, b_{n}\right]$ are defined as follows:

$$
\begin{aligned}
& \min \left[b_{1}, b_{2}, \cdots, b_{n}\right]=\left[\underset{i=1, \cdots n}{\wedge} \underset{i=1, \cdots \cdot n}{\wedge} b_{i}, \wedge b_{i}, \cdots, \underset{i=1, \cdots n}{\wedge} b_{i}\right], \\
& \max \left[b_{1}, b_{2}, \cdots, b_{n}\right]=\left[\underset{i=1, \cdots n}{\vee} b_{i=1, \cdots n}, \underset{i=n}{\vee} b_{i}, \cdots, \underset{i=1, \cdots \cdot n}{\vee} b_{i}\right]
\end{aligned}
$$

$\min \left[b_{1}, b_{2}, \cdots, b_{n}\right]$ and $\max \left[b_{1}, b_{2}, \cdots, b_{n}\right]$ are the lower and upper bounds of $\left[b_{1}, b_{2}, \cdots, b_{n}\right] \quad, \quad$ respectively. $\quad$ For $\quad$ example, $\min [0.3,0.8]=[0.3,0.3] \quad, \quad$ and $\max [0.3,0.8]=[0.8,0.8]$.

In the following, we consider four types of focus points. 
Active focus point: The state (scenario) with the higher possibility degree and the higher satisfaction level is obtained as

$$
x_{1}(a)=\underset{x \in S}{\arg \max } \min [\pi(x), u(x, a)] .
$$

$x_{1}(a)$ is called an active focus point of an alternative $a . \underset{x \in S}{\arg \max } \min [\pi(x), u(x, a)]$ represents an element of $S$ which maximizes $\min [\pi(x), u(x, a)]$ with $x \in S$. Because $\min [\pi(x), u(x, a)]$ represents the lower bound of the vector $[\pi(x), u(x, a)]$, maximizing $\min [\pi(x), u(x, a)]\left(\max _{x \in S} \min [\pi(x), u(x, a)]\right)$ will increase the possibility degree and the satisfaction level simultaneously. Therefore, $\underset{x \in S}{\arg \max } \min [\pi(x), u(x, a)]$ is for seeking the state that has the higher possibility degree and the higher satisfaction level.

In order to facilitate understanding (3), let us give an example. For four states $S=\left\{x_{1}, x_{2}, x_{3}, x_{4}\right\}$, we have $\pi\left(x_{1}\right)=0.1, \pi\left(x_{2}\right)=0.3, \pi\left(x_{3}\right)=1.0, \pi\left(x_{4}\right)=0.6$, $u\left(x_{1}, a\right)=0.6, u\left(x_{2}, a\right)=0.2, u\left(x_{3}, a\right)=0.3 \quad$ and $u\left(x_{4}, a\right)=0.8 .[\pi(x), u(x, a)]$, $x \in S$ are four vectors: $[0.1,0.6],[0.3,0.2],[1.0,0.3]$ and $[0.6,0.8]$ represented by $A, B$, $C$ and $D$, respectively (shown in Fig. 1.). $\min [\pi(x), u(x, a)]$ transfers $A, B, C$ and $D$ into $A^{\prime}$, $B^{\prime}, C^{\prime}$ and $D^{\prime}$, which are $[0.1,0.1],[0.2,0.2],[0.3,0.3]$ and $[0.6,0.6]$, respectively. $\max _{x \in S} \min [\pi(x), u(x, a)]$ is $\max ([0.1,0.1],[0.2,0.2],[0.3,0.3],[0.6,0.6])=[0.6,0.6]$ which corresponds to $D^{\prime}$. Thus, $\underset{x \in S}{\arg \max } \min [\pi(x), u(x, a)] \quad$ is $x_{4}$. It is obvious that $x_{4}$ have a higher possibility degree (0.6) and a higher satisfaction level (0.8).

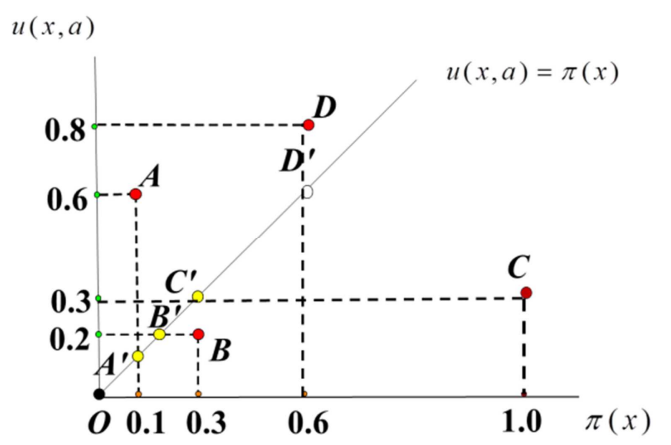

Fig.1 The explanation of the formula (3) 
Passive focus point: The state (scenario) with the higher possibility degree and the lower satisfaction level is obtained as

$$
x_{2}(a)=\underset{x \in S}{\arg \min } \max [1-\pi(x), u(x, a)] .
$$

$x_{2}(a)$ is called a passive focus point of an alternative $a$.

Apprehensive focus point: The state (scenario) with the lower possibility degree and the lower satisfaction level is obtained as

$$
x_{3}(a)=\underset{x \in S}{\arg \min } \max [\pi(x), u(x, a)] .
$$

$x_{3}(a)$ is called an apprehensive focus point of an alternative $a$.

Daring focus point: The state (scenario) with the lower possibility degree and the higher satisfaction level is obtained as

$$
x_{4}(a)=\underset{x \in S}{\arg \min } \max [\pi(x), 1-u(x, a)] .
$$

$x_{4}(a)$ is called a daring focus point of an alternative $a$.

Comments: From (3)-(6), we know that no other $[\pi(x), u(x, a)]$ satisfies $\pi(x)>\pi\left(x_{1}(a)\right)$ and $u(x, a)>u\left(x_{1}(a), a\right) ; \quad$ or $\quad \pi(x)>\pi\left(x_{2}(a)\right) \quad$ and $\quad u(x, a)<u\left(x_{2}(a), a\right) ; \quad$ or $\pi(x)<\pi\left(x_{3}(a)\right) \quad$ and $\quad u(x, a)<u\left(x_{3}(a), a\right) \quad ; \quad$ or $\quad \pi(x)<\pi\left(x_{4}(a)\right) \quad$ and $u(x, a)>u\left(x_{4}(a), a\right)$. In other words, there is no state which has a higher possibility degree and a higher satisfaction degree than the active focus point; a higher possibility degree and a lower satisfaction level than the passive focus point; a lower possibility degree and a lower satisfaction level than the apprehensive focus point; a lower possibility degree and a higher satisfaction level than the daring focus point.

For one alternative, more than one state might exist as one type of focus point. We denote the sets of four types of focus points of an alternative $a$ as $X_{1}(a), X_{2}(a), X_{3}(a)$ and $X_{4}(a)$, respectively.

In a one-shot decision problem, a decision maker contemplates that the focus points are the most appropriate scenarios for him/her. After determining the focus points of each alternative, the decision maker will make a decision only based on the focus points and chooses the optimal alternative which can bring about the highest satisfaction level once its focus point comes true. The four kinds of optimal alternatives are obtained as follows: 


$$
\begin{aligned}
& a_{1}^{*}=\underset{a \in A}{\arg \max } \max _{x_{1}(a) \in X_{1}(a)} u\left(x_{1}(a), a\right), \\
& a_{2}^{*}=\underset{a \in A}{\arg \max } \min _{x_{2}(a) \in X_{2}(a)} u\left(x_{2}(a), a\right), \\
& a_{3}^{*}=\underset{a \in A}{\arg \max } \min _{x_{3}(a) \in X_{3}(a)} u\left(x_{3}(a), a\right), \\
& a_{4}^{*}=\underset{a \in A}{\arg \max } \max _{x_{4}(a) \in X_{4}(a)} u\left(x_{4}(a), a\right) .
\end{aligned}
$$

In the case where multiple active focus points of an alternative $a$ exist, $\max _{x_{1}(a) \in X_{1}(a)} u\left(x_{1}(a), a\right)$ is used to represent the highest satisfaction level amongst all active focus points of $a$. It reflects an optimistic attitude of a decision maker whereas $\min _{x_{2}(a) \in X_{2}(a)} u\left(x_{2}(a), a\right)$ describes a conservative attitude of a decision maker. $a_{1}^{*}, a_{2}^{*}, a_{3}^{*}$ and $a_{4}^{*}$ are called optimal active, passive, apprehensive and daring alternatives, respectively. Setting $x_{1}^{*}=x_{1}\left(a_{1}^{*}\right), x_{2}^{*}=x_{2}\left(a_{2}^{*}\right)$, $x_{3}^{*}=x_{3}\left(a_{3}^{*}\right)$ and $x_{4}^{*}=x_{4}\left(a_{4}^{*}\right), x_{1}^{*}, x_{2}^{*}, x_{3}^{*}$ and $x_{4}^{*}$ are called optimal active, passive, apprehensive and daring focus points, respectively.

\subsection{General solutions of focus points and optimal alternatives}

If $S$ and $A$ are nonempty finite sets, there are always solutions of (3)-(10). For the continuous cases, let us consider the solutions of (3)-(10) with the following conditions.

Basic Assumptions: In the following parts, we suppose

(1) The sets of states and alternatives are $S=[l, h]$ and $A=\left[a_{l}, a_{h}\right]$, respectively.

(2) $\pi(x)$ is a strictly quasi-concave continuous function (see the definition in the book (Madden, 1986)), $\exists c \in(l, h), \pi(c)=1, \pi(l)=0$ and $\pi(h)=0$.

(3) $v(x, a)$ is continuous and strictly quasi-concave in $x$.

Clearly, $\pi(x)$ is strictly increasing within $[l, c]$ and strictly decreasing within $[c, h]$. $u(x, a)$ is continuous and strictly quasi-concave in $x . \forall a \quad u(x, a)$ attains its maximum at a unique state $\hat{x}(a)=\underset{x \in[l, h]}{\arg \max } u(x, a)$ and is strictly increasing within $[l, \hat{x}(a)]$ for $\hat{x}(a) \neq l$ and strictly decreasing within $[\hat{x}(a), h]$ for $\hat{x}(a) \neq h$.

We have the following theorems.

Theorem 1. The active focus point of an alternative $a, x_{1}(a)$, is as follows: 
(1) if $u(\hat{x}(a), a) \leq \pi(\hat{x}(a))$, then $x_{1}(a)=\hat{x}(a)$;

(2) if $u(\hat{x}(a), a) \geq \pi(\hat{x}(a))$ and $\hat{x}(a) \leq c$, then $x_{1}(a)=x_{o l}(a)$;

(3) if $u(\hat{x}(a), a) \geq \pi(\hat{x}(a))$ and $\hat{x}(a) \geq c$, then $x_{1}(a)=x_{o u}(a)$.

$x_{o l}(a)$ and $x_{o u}(a)$ are the horizontal coordinates of the intersections of $u(x, a)$ and $\pi(x)$ within $[\hat{x}(a), c]$ and $[c, \hat{x}(a)]$, respectively.

\section{Proof.}

(1) We have

$$
\max _{x \in[l, h]} \min [\pi(x), u(x, a)] \leq \max _{x \in[l, h]}[u(x, a), u(x, a)]=[u(\hat{x}(a), a), u(\hat{x}(a), a)] .
$$

$\min [\pi(x), u(x, a)]$ attains its maximum $[u(\hat{x}(a), a), u(\hat{x}(a), a)]$ if and only if $x=\hat{x}(a)$. It means $x_{1}(a)=\hat{x}(a)$.

(2) First, let us consider the cases satisfying $\hat{x}(a) \neq c$. Since $\pi(\hat{x}(a)) \leq u(\hat{x}(a), a)$ and $u(c, a)<\pi(c)=1$ and $u(x, a)$ is strictly decreasing and $\pi(x)$ is strictly increasing within $[\hat{x}(a), c], u(x, a)$ and $\pi(x)$ have a unique intersection within $[\hat{x}(a), c)$. The horizontal coordinate of this intersection is denoted as $x_{o l}(a) . \forall x \in\left[l, x_{o l}(a)\right), \pi(x)$ is strictly increasing so that

$$
\min [\pi(x), u(x, a)] \leq[\pi(x), \pi(x)]<\left[\pi\left(x_{o l}(a)\right), \pi\left(x_{o l}(a)\right)\right] .
$$

$\forall x \in\left(x_{o l}(a), h\right], u(x, a)$ is a strictly decreasing function of $x$ so that

$$
\min [\pi(x), u(x, a)] \leq[u(x, a), u(x, a)]<\left[u\left(x_{o l}(a), a\right), u\left(x_{o l}(a), a\right)\right] .
$$

Recalling $\pi\left(x_{o l}(a)\right)=u\left(x_{o l}(a), a\right)$, we have

$$
x_{1}(a)=\underset{x \in[l, h]}{\arg \max } \min \left[(\pi(x), u(x, a)]=x_{o l}(a) .\right.
$$

We can directly check that (14) also holds for $\hat{x}(a)=c$.

(3) Similarly, we have $x_{1}(a)=x_{o u}(a) . x_{o u}(a)$ is the horizontal coordinate of the unique intersection of $u(x, a)$ and $\pi(x)$ within $[c, \hat{x}(a)]$.

Theorem 2. The passive focus point of an alternative $a, x_{2}(a)$, is as follows:

(1) if $u(\hat{x}(a), a) \geq 1-\pi(\hat{x}(a))$, then $x_{2}(a)=\underset{x \in\left\{x_{p l}(a), x_{p u}(a)\right\}}{\arg \min } u(x, a)$;

(2) if $u(\hat{x}(a), a) \leq 1-\pi(\hat{x}(a))$ and $\hat{x}(a)>c$, then $x_{2}(a)=x_{p l}(a)$; 
(3) if $u(\hat{x}(a), a) \leq 1-\pi(\hat{x}(a))$ and $\hat{x}(a)<c$, then $\quad x_{2}(a)=x_{p u}(a)$.

$x_{p l}(a)$ and $x_{p u}(a)$ are the horizontal coordinates of the intersections of $u(x, a)$ and $1-\pi(x)$ within $[l, \min (\hat{x}(a), c)]$ and $[\max (\hat{x}(a), c), h]$, respectively.

\section{Proof.}

(1) First, let us consider the cases satisfying $\hat{x}(a) \neq l, \hat{x}(a) \neq h$ and $u(\hat{x}(a), a) \neq 1-\pi(\hat{x}(a))$. Since $u(l, a)<1-\pi(l)=1$ and $u(\hat{x}(a), a)>1-\pi(\hat{x}(a))$ and $u(c, a)>1-\pi(c)=0$ and $u(x, a)$ is strictly increasing continuous in $x$ and $1-\pi(x)$ is strictly decreasing continuous within $[l, \min (\hat{x}(a), c)], u(x, a)$ and $1-\pi(x)$ have a unique intersection within $(l, \min (\hat{x}(a), c))$. Similarly, it is easy to know that they have a unique intersection within $(\max (\hat{x}(a), c), h)$. The horizontal coordinates of these two intersections are $x_{p l}(a)$ and $x_{p u}(a)$, respectively. $\forall x \in\left[l, x_{p l}(a)\right), 1-\pi(x)$ is strictly decreasing so that

$$
\max [1-\pi(x), u(x, a)] \geq[1-\pi(x), 1-\pi(x)]>\left[1-\pi\left(x_{p l}(a)\right), 1-\pi\left(x_{p l}(a)\right)\right] .
$$

$\forall x \in\left(x_{p l}(a), \min (\hat{x}(a), c)\right], u(x, a)$ is a strictly increasing function of $x$ so that

$$
\max [1-\pi(x), u(x, a)] \geq[u(x, a), u(x, a)]>\left[u\left(x_{p l}(a), a\right), u\left(x_{p l}(a), a\right)\right] .
$$

Recalling $1-\pi\left(x_{p l}(a)\right)=u\left(x_{p l}(a), a\right)$, we have

$$
\underset{x \in[l, \min (\hat{x}(a), c)]}{\arg \min } \max [1-\pi(x), u(x, a)]=x_{p l}(a) .
$$

Similarly, we have

$$
\underset{x \in[\max (\hat{x}(a), c), h]}{\arg \min } \max [1-\pi(x), u(x, a)]=x_{p u}(a) .
$$

If $\hat{x}(a) \geq c$, the interval $[\min (\hat{x}(a), c), \max (\hat{x}(a), c)]$ becomes $[c, \hat{x}(a)]$. Since $u(x, a)$ is strictly increasing within $\left[x_{p l}(a), \hat{x}(a)\right]$, we have

$$
\begin{aligned}
& \min _{x \in[c, \hat{x}(a)]} \max [1-\pi(x), u(x, a)] \geq \min _{x \in[c, \hat{x}(a)]}[u(x, a), u(x, a)] \\
& =[u(c, a), u(c, a)]>\left[u\left(x_{p l}(a), a\right), u\left(x_{p l}(a), a\right)\right] .
\end{aligned}
$$

Similarly, if $\hat{x}(a)<c$, the interval $[\min (\hat{x}(a), c), \max (\hat{x}(a), c)]$ becomes $[\hat{x}(a), c]$. Since 
$u(x, a)$ is strictly decreasing within $\left[\hat{x}(a), x_{p u}(a)\right]$, we have

$$
\min _{x \in[\hat{x}(a), c]} \max [1-\pi(x), u(x, a)]>\left[u\left(x_{p u}(a), a\right), u\left(x_{p u}(a), a\right)\right] .
$$

From (17) to (20), we know

$$
x_{2}(a)=\underset{x \in[l, h]}{\arg \min } \max [1-\pi(x), u(x, a)]=\underset{x \in\left\{x_{p l}(a), x_{p u}(a)\right\}}{\arg \min } u(x, a) .
$$

It is easy to check that (21) also holds for the case $u(\hat{x}(a), a)=1-\pi(\hat{x}(a))$ or $\hat{x}(a)=l$ or $\hat{x}(a)=h$.

Likewise, we can prove Theorem 2(2) and 2(3).

Corollary 3. Suppose $u(\hat{x}(a), a) \geq 1-\pi(\hat{x}(a))$. The passive focus point $x_{2}(a)$ is as follows:

(1) if $\hat{x}(a)>c$ and $\exists x_{0} \in(c, \hat{x}(a)] \quad u\left(x_{0}, a\right)=1-\pi\left(x_{0}\right)$ holds, then

$$
x_{2}(a)=\underset{x \in\left\{x_{p l}(a), x_{p u}(a)\right\}}{\arg \min } u(x, a)=x_{p l}(a)
$$

(2) if $\hat{x}(a)<c$ and $\exists x_{0} \in[\hat{x}(a), c) \quad u\left(x_{0}, a\right)=1-\pi\left(x_{0}\right)$ holds, then

$$
x_{2}(a)=\underset{x \in\left\{x_{p l}(a), x_{p u}(a)\right\}}{\arg \min } u(x, a)=x_{p u}(a) .
$$

\section{Proof.}

(1) Since $1-\pi(x)$ is strictly increasing within $[c, h]$ and $c<x_{0} \leq x_{p u}(a) \leq h$, $1-\pi\left(x_{p u}(a)\right) \geq 1-\pi\left(x_{0}\right)$ holds. Since $u(x, a)$ is strictly increasing within $x \in[l, \hat{x}(a)]$ and $l \leq x_{p l}(a) \leq c<x_{0} \leq \hat{x}(a), u\left(x_{0}, a\right)>u\left(x_{p l}(a), a\right)$ holds. Therefore, we have

$$
u\left(x_{p u}(a), a\right)=1-\pi\left(x_{p u}(a)\right) \geq 1-\pi\left(x_{0}\right)=u\left(x_{0}, a\right)>u\left(x_{p l}(a), a\right) .
$$

From Theorem 2(1), we know $x_{2}(a)=x_{p l}(a)$.

(2) Likewise, we can prove Corollary 3(2).

\section{Lemma 4.}

(1) $\forall a_{1}, a_{2} \in A \quad u\left(x_{p l}\left(a_{1}\right), a_{1}\right)<u\left(x_{p l}\left(a_{2}\right), a_{2}\right)$ holds if and only if $x_{p l}\left(a_{1}\right)>x_{p l}\left(a_{2}\right)$ holds; and $u\left(x_{p l}\left(a_{1}\right), a_{1}\right)=u\left(x_{p l}\left(a_{2}\right), a_{2}\right)$ holds if and only if $x_{p l}\left(a_{1}\right)=x_{p l}\left(a_{2}\right)$ holds. 
(2) $\forall a_{1}, a_{2} \in A \quad u\left(x_{p u}\left(a_{1}\right), a_{1}\right)<u\left(x_{p u}\left(a_{2}\right), a_{2}\right)$ holds if and only if $x_{p u}\left(a_{1}\right)<x_{p u}\left(a_{2}\right)$ holds; and $u\left(x_{p u}\left(a_{1}\right), a_{1}\right)=u\left(x_{p u}\left(a_{2}\right), a_{2}\right)$ holds if an only if $x_{p u}\left(a_{1}\right)=x_{p u}\left(a_{2}\right)$ holds.

\section{Corollary 5.}

(1) Assume that $u\left(\hat{x}\left(a_{i}\right), a_{i}\right) \geq 1-\pi\left(\hat{x}\left(a_{i}\right)\right)$ or $u\left(\hat{x}\left(a_{i}\right), a_{i}\right) \leq 1-\pi\left(\hat{x}\left(a_{i}\right)\right)$ with $\hat{x}\left(a_{i}\right)>c$, $i=1,2$. If $u\left(x, a_{1}\right) \leq u\left(x, a_{2}\right)$ holds for any $x \in\left[l, \min \left(\hat{x}\left(a_{1}\right), \hat{x}\left(a_{2}\right)\right)\right]$, then $u\left(x_{p l}\left(a_{1}\right), a_{1}\right) \leq u\left(x_{p l}\left(a_{2}\right), a_{2}\right) \quad$ holds; $\quad$ and $\quad$ if $\quad u\left(x, a_{1}\right)<u\left(x, a_{2}\right)$ holds for any $x \in\left[l, \min \left(\hat{x}\left(a_{1}\right), \hat{x}\left(a_{2}\right)\right)\right]$, then $u\left(x_{p l}\left(a_{1}\right), a_{1}\right)<u\left(x_{p l}\left(a_{2}\right), a_{2}\right)$ holds.

(2) Assume that $u\left(\hat{x}\left(a_{i}\right), a_{i}\right) \geq 1-\pi\left(\hat{x}\left(a_{i}\right)\right)$ or $u\left(\hat{x}\left(a_{i}\right), a_{i}\right) \leq 1-\pi\left(\hat{x}\left(a_{i}\right)\right)$ with $\hat{x}\left(a_{i}\right)<c$, $i=1,2$. If $u\left(x, a_{1}\right) \leq u\left(x, a_{2}\right)$ holds for any $x \in\left[\max \left(\hat{x}\left(a_{1}\right), \hat{x}\left(a_{2}\right)\right), h\right]$, then $u\left(x_{p u}\left(a_{1}\right), a_{1}\right) \leq u\left(x_{p u}\left(a_{2}\right), a_{2}\right) \quad$ holds; $\quad$ and $\quad$ if $u\left(x, a_{1}\right)<u\left(x, a_{2}\right)$ holds for any $x \in\left[\max \left(\hat{x}\left(a_{1}\right), \hat{x}\left(a_{2}\right)\right), h\right]$, then $u\left(x_{p u}\left(a_{1}\right), a_{1}\right)<u\left(x_{p u}\left(a_{2}\right), a_{2}\right)$ holds.

Lemma 6. The apprehensive focus point of an alternative $a, x_{3}(a)$, is as follows:

$$
x_{3}(a)=\underset{x \in\{l, h\}}{\arg \min } u(x, a) .
$$

Proof. $u(x, a)$ is a strictly quasi-concave function in $x$ so that $\min _{x \in[l, h]} \max [\pi(x), u(x, a)] \geq \min _{x \in[l, h]}[u(x, a), u(x, a)]=[u(l, a), u(l, a)] \wedge[u(h, a), u(h, a)]$,

where the equality holds if and only if $x=\underset{x \in\{l, h\}}{\arg \min } u(x, a)$ because $\pi(l)=\pi(h)=0$.

Therefore, (23) holds.

Lemma 7. The daring focus point of an alternative $a, x_{4}(a)$, is as follows:

(1) if $1-u(\hat{x}(a), a) \geq \pi(\hat{x}(a))$, then $x_{4}(a)=\hat{x}(a)$;

(2) if $1-u(\hat{x}(a), a) \leq \pi(\hat{x}(a))$, then $x_{4}(a)=\underset{x \in\left\{x_{d l}(a), x_{d u}(a)\right\}}{\arg \max } u(x, a)$.

$x_{d l}(a)$ and $x_{d u}(a)$ are the horizontal coordinates of the intersections of $1-u(x, a)$ and $\pi(x)$ within $[l, \min (\hat{x}(a), c)]$ and $[\max (\hat{x}(a), c), h]$, respectively. 
Proof. (1) We have

$$
\begin{aligned}
\min _{x \in[l, h]} \max [\pi(x), 1-u(x, a)] & \geq \min _{x \in[l, h]}[1-u(x, a), 1-u(x, a)] \\
& =[1-u(\hat{x}(a), a), 1-u(\hat{x}(a), a)],
\end{aligned}
$$

where the equation holds if and only if $x=\hat{x}(a)$. That is, $x_{4}(a)=\hat{x}(a)$.

(2) Referring to the proof of Theorem 2(1), we have

$$
x_{4}(a)=\underset{x \in[l, h]}{\arg \min } \max [\pi(x), 1-u(x, a)]=\underset{x \in\left\{x_{d l}(a), x_{d u}(a)\right\}}{\arg \max } u(x, a) .
$$

Theorem 8. If there is an closed interval $G \subseteq\left[a_{l}, a_{h}\right]$ and $\forall a \in G \quad x_{o l}(a)$ exists, then $x_{o l}(a), \pi\left(x_{o l}(a)\right), u\left(x_{o l}(a), a\right)$ are uniformly continuous within $G . x_{o u}(a), \pi\left(x_{o u}(a)\right)$, $u\left(x_{o u}(a), a\right), x_{p l}(a), \pi\left(x_{p l}(a)\right), u\left(x_{p l}(a), a\right), x_{p u}(a), \pi\left(x_{p u}(a)\right), u\left(x_{p u}(a), a\right)$, $x_{d l}(a), \quad \pi\left(x_{d l}(a)\right), \quad u\left(x_{d l}(a), a\right), \quad x_{d u}(a), \quad \pi\left(x_{d u}(a)\right)$ and $u\left(x_{d u}(a), a\right)$ are also uniformly continuous within their corresponding closed intervals, respectively.

Proof. $\forall a_{1}, a_{2} \in G$, for simplicity we assume $x_{o l}\left(a_{1}\right) \leq x_{o l}\left(a_{2}\right)$. Since $u\left(x_{o l}\left(a_{2}\right), a\right)$ is a continuous function of $a$ within $\left[a_{l}, a_{h}\right], u\left(x_{o l}\left(a_{2}\right), a\right)$ is a uniformly continuous function. That is, $\forall \varepsilon>0, \exists \delta>0$ such that $\left|a_{1}-a_{2}\right|<\delta$ implies

$$
\left|u\left(x_{o l}\left(a_{2}\right), a_{1}\right)-u\left(x_{o l}\left(a_{2}\right), a_{2}\right)\right|<\varepsilon .
$$

$\hat{x}\left(a_{1}\right) \leq x_{o l}\left(a_{1}\right) \leq x_{o l}\left(a_{2}\right)$ leads to $u\left(x_{o l}\left(a_{1}\right), a_{1}\right) \geq u\left(x_{o l}\left(a_{2}\right), a_{1}\right)$ so that we have

$$
u\left(x_{o l}\left(a_{1}\right), a_{1}\right)-u\left(x_{o l}\left(a_{2}\right), a_{2}\right) \geq u\left(x_{o l}\left(a_{2}\right), a_{1}\right)-u\left(x_{o l}\left(a_{2}\right), a_{2}\right) .
$$

Recalling $x_{o l}\left(a_{1}\right) \leq x_{o l}\left(a_{2}\right) \leq c$, we have

$$
u\left(x_{o l}\left(a_{1}\right), a_{1}\right)-u\left(x_{o l}\left(a_{2}\right), a_{2}\right)=\pi\left(x_{o l}\left(a_{1}\right)\right)-\pi\left(x_{o l}\left(a_{2}\right)\right) \leq 0 .
$$

Therefore,

$$
\begin{aligned}
& \left|\pi\left(x_{o l}\left(a_{1}\right)\right)-\pi\left(x_{o l}\left(a_{2}\right)\right)\right|=\left|u\left(x_{o l}\left(a_{1}\right), a_{1}\right)-u\left(x_{o l}\left(a_{2}\right), a_{2}\right)\right| \\
& \leq\left|u\left(x_{o l}\left(a_{2}\right), a_{1}\right)-u\left(x_{o l}\left(a_{2}\right), a_{2}\right)\right|<\varepsilon .
\end{aligned}
$$

We know that $\pi\left(x_{o l}(a)\right), u\left(x_{o l}(a), a\right)$ are uniformly continuous functions within $G$ so that $x_{o l}(a)$ is uniformly continuous within $G$. Likewise, we have the same conclusions for $x_{\text {ou }}(a), \pi\left(x_{\text {ou }}(a)\right), \quad u\left(x_{\text {ou }}(a), a\right), \quad x_{p l}(a), \pi\left(x_{p l}(a)\right), \quad u\left(x_{p l}(a), a\right), \quad x_{p u}(a)$, 


$$
\begin{aligned}
& \pi\left(x_{p u}(a)\right), u\left(x_{p u}(a), a\right), x_{d l}(a), \pi\left(x_{d l}(a)\right), u\left(x_{d l}(a), a\right), x_{d u}(a), \pi\left(x_{d u}(a)\right) \text { and } \\
& u\left(x_{d u}(a), a\right)
\end{aligned}
$$

\section{Lemma 9.}

(1) $u\left(x_{1}(a), a\right)=\max _{x \in[l, h]} \min (\pi(x), u(x, a))$,

(2) $u\left(x_{2}(a), a\right)=\min _{x \in[l, h]} \max (1-\pi(x), u(x, a))$,

(3) $u\left(x_{3}(a), a\right)=\min _{x \in[l, h]} \max (\pi(x), u(x, a))$,

(4) $u\left(x_{4}(a), a\right)=\max _{x \in[l, h]} \min (1-\pi(x), u(x, a))$.

Theorem 10 (Existence Theorem). If the basic assumptions (1), (2) and (3) hold, then $a_{1}^{*}, a_{2}^{*}$, $a_{3}^{*}, a_{4}^{*}, x_{1}\left(a_{1}^{*}\right), x_{2}\left(a_{2}^{*}\right), x_{3}\left(a_{3}^{*}\right)$ and $x_{4}\left(a_{4}^{*}\right)$ always exist and they satisfy the following relations:

(1) $u\left(x_{1}\left(a_{1}^{*}\right), a_{1}^{*}\right)=\max _{a \in\left[a_{l}, a_{h}\right]} \max _{x \in[l, h]} \min (\pi(x), u(x, a))$,

(2) $u\left(x_{2}\left(a_{2}^{*}\right), a_{2}^{*}\right)=\max _{a \in\left[a_{l}, a_{h}\right]} \min _{x \in[l, h]} \max (1-\pi(x), u(x, a))$,

(3) $u\left(x_{3}\left(a_{3}^{*}\right), a_{3}^{*}\right)=\max _{a \in\left[a_{l}, a_{h}\right]} \min _{x \in[l, h]} \max (\pi(x), u(x, a))$,

(4) $u\left(x_{4}\left(a_{4}^{*}\right), a_{4}^{*}\right)=\max _{a \in\left[a_{l}, a_{h}\right]} \max _{x \in[l, h]} \min (1-\pi(x), u(x, a))$.

Proof. Set $g(x, a)=\min (\pi(x), u(x, a))$. Since $u(x, a)$ is continuous on $[l, h] \times\left[a_{l}, a_{h}\right]$ and $\pi(x)$ is continuous on $[l, h], g(x, a)$ is continuous on $[l, h] \times\left[a_{l}, a_{h}\right]$. Using Berge maximum theorem and Lemma 9(1), we know $u\left(x_{1}(a), a\right)=\max _{x \in[l, h]} \min (\pi(x), u(x, a))$ is continuous so that $a_{1}^{*}$ and $x_{1}\left(a_{1}^{*}\right)$ exist and satisfy Theorem 10(1). Likewise, we can prove Theorem 10(1), 10(2), 10(3) and 10(4).

\section{Lemma 11.}

(1) $u\left(x_{1}\left(a_{1}^{*}\right), a_{1}^{*}\right)=\max _{x \in[l, h]} \min \left(\pi(x), \max _{a \in\left[a_{l}, a_{h}\right]} u(x, a)\right)$, 
(2) $u\left(x_{4}\left(a_{4}^{*}\right), a_{4}^{*}\right)=\max _{x \in[l, h]} \min \left(1-\pi(x), \max _{a \in\left[a_{l}, a_{h}\right]} u(x, a)\right)$.

Proof. From Theorem 10(1), we have

$$
\begin{aligned}
& u\left(x_{1}\left(a_{1}^{*}\right), a_{1}^{*}\right)=\max _{a \in\left[a_{l}, a_{h}\right]} \max _{x \in[l, h]} \min (\pi(x), u(x, a)) \\
& =\max _{x \in[l, h]} \max _{a \in\left[a_{l}, a_{h}\right]} \min (\pi(x), u(x, a))=\max _{x \in[l, h]} \min \left(\pi(x), \max _{a \in\left[a_{l}, a_{h}\right]} u(x, a)\right) .
\end{aligned}
$$

From Theorem 10(4), we have

$$
\begin{aligned}
u\left(x_{4}\left(a_{4}^{*}\right), a_{4}^{*}\right) & =\max _{a \in\left[a_{l}, a_{h}\right]} \max _{x \in[l, h]} \min (1-\pi(x), u(x, a)) \\
& =\max _{x \in[l, h]} \min \left(1-\pi(x), \max _{a \in\left[a_{l}, a_{h}\right]} u(x, a)\right) .
\end{aligned}
$$

Let us consider $v(x, a)$ is quasi-convex continuous in $x$ and quasi-concave continuous in $a$ so that $u(x, a)$ is quasi-convex continuous in $x$ and quasi-concave continuous in $a$. We have the following theorem.

Theorem 12. If $u(x, a)$ is quasi-convex continuous in $x$ and quasi-concave continuous in $a$, then we have

$$
\max _{a \in\left[a_{l}, a_{h}\right]} \min _{x \in[l, h]} \max (1-\pi(x), u(x, a))=\min _{x \in[l, h]} \max \left(1-\pi(x), \max _{a \in\left[a_{l}, a_{h}\right]} u(x, a)\right) .
$$

Proof. Since $\pi(x)$ is strictly quasi-concave, $1-\pi(x)$ is strictly quasi-convex. For any $x_{1}$ and $x_{2}$, we have

$$
1-\pi\left(\lambda x_{1}+(1-\lambda) x_{2}\right)<\max \left(1-\pi\left(x_{1}\right), 1-\pi\left(x_{2}\right)\right), \quad \forall \lambda \in(0,1) .
$$

$u(x, a)$ is quasi-convex in $x$ so that for any $x_{1}, x_{2}$ we have

$$
u\left(\lambda x_{1}+(1-\lambda) x_{2}, a\right) \leq \max \left(u\left(x_{1}, a\right), u\left(x_{2}, a\right)\right), \quad \forall \lambda \in(0,1) .
$$

Setting $f(x, a)=\max (1-\pi(x), u(x, a))$ and considering (34) and (35), we have

$$
\begin{aligned}
& f\left(\lambda x_{1}+(1-\lambda) x_{2}, a\right)=\max \left(1-\pi\left(\lambda x_{1}+(1-\lambda) x_{2}\right), u\left(\lambda x_{1}+(1-\lambda) x_{2}, a\right)\right) \\
& \leq \max \left(\max \left(1-\pi\left(x_{1}\right), 1-\pi\left(x_{2}\right)\right), \max \left(u\left(x_{1}, a\right), u\left(x_{2}, a\right)\right)\right) \\
& =\max \left(\max \left(1-\pi\left(x_{1}\right), u\left(x_{1}, a\right)\right), \max \left(1-\pi\left(x_{2}\right), u\left(x_{2}, a\right)\right)\right) \\
& =\max \left(f\left(x_{1}, a\right), f\left(x_{2}, a\right)\right),
\end{aligned}
$$

which means $f(x, a)$ is quasi-convex in $x . u(x, a)$ is a quasi-concave function of $a$, that is,

$$
u\left(x, \lambda a_{1}+(1-\lambda) a_{2}\right) \geq \min \left(u\left(x, a_{1}\right), u\left(x, a_{2}\right)\right), \quad \forall \lambda \in(0,1) .
$$

Considering (37), we have

$$
f\left(x, \lambda a_{1}+(1-\lambda) a_{2}\right)=\max \left(1-\pi(x), u\left(x, \lambda a_{1}+(1-\lambda) a_{2}\right)\right)
$$




$$
\begin{aligned}
& \geq \max \left(1-\pi(x), \min \left(u\left(x, a_{1}\right), u\left(x, a_{2}\right)\right)\right) \\
& =\min \left(\max \left(1-\pi(x), u\left(x, a_{1}\right)\right), \max \left(1-\pi(x), u\left(x, a_{2}\right)\right)\right) \\
& =\min \left(f\left(x, a_{1}\right), f\left(x, a_{2}\right)\right),
\end{aligned}
$$

which means $f(x, a)$ is quasi-concave in $a$. Since $u(x, a)$ and $\pi(x)$ are continuous, $f(x, a)$ is a continuous function. According to Sion's minimax theorem (Sion, 1958), we have

$$
\begin{aligned}
& \max _{a \in\left[a_{l}, a_{h}\right]} \min _{x \in[l, h]} \max (1-\pi(x), u(x, a))=\min _{x \in[l, h]} \max _{a \in\left[a_{l}, a_{h}\right]} \max (1-\pi(x), u(x, a)) \\
& =\min _{x \in[l, h]} \max \left(1-\pi(x), \max _{a \in\left[a_{l}, a_{h}\right]} u(x, a)\right) .
\end{aligned}
$$

\section{Theorem 13.}

(1) If $\max _{a \in\left[a_{l}, a_{h}\right]} u(x, a)$ is strictly increasing, then the unique optimal active focus point $x_{1}^{*}$ satisfies $\pi(x)=\max _{a \in\left[a_{l}, a_{h}\right]} u(x, a), x \in(c, h)$ and $a_{1}^{*}=\underset{a \in\left[a_{l}, a_{h}\right]}{\arg \max } u\left(x_{1}^{*}, a\right)$.

(2) If $\max _{a \in\left[a_{l}, a_{h}\right]} u(x, a)$ is strictly increasing and $\max _{a \in\left[a_{l}, a_{h}\right]} u(h, a)=1$, then the unique optimal daring focus point is $x_{4}^{*}=h$ and $a_{4}^{*}=\underset{a \in\left[a_{l}, a_{h}\right]}{\arg \max } u\left(x_{4}^{*}, a\right)$.

(3) If $\max _{a \in\left[a_{l}, a_{h}\right]} u(x, a)$ is strictly decreasing, then the unique optimal active focus point $x_{1}^{*}$ satisfies $\pi(x)=\max _{a \in\left[a_{l}, a_{h}\right]} u(x, a), x \in(l, c)$ and $a_{1}^{*}=\underset{a \in\left[a_{l}, a_{h}\right]}{\arg \max } u\left(x_{1}^{*}, a\right)$.

(4) If $\max _{a} u(x, a)$ is strictly decreasing and $\max _{a} u(l, a)=1$, then the unique optimal daring focus point is $x_{4}^{*}=l$ and $a_{4}^{*}=\underset{a \in\left[a_{l}, a_{h}\right]}{\arg \max } u\left(x_{4}^{*}, a\right)$.

\section{Proof.}

(1) It follows from Berge maximum theorem that $\max _{a \in\left[a_{l}, a_{h}\right]} u(x, a)$ is continuous because $u(x, a)$ is continuous. Since $\max _{a \in\left[a_{l}, a_{h}\right]} u(c, a)<\pi(c)=1$ and $\max _{a \in\left[a_{l}, a_{h}\right]} u(h, a)>\pi(h)=0$ and $\max _{a \in\left[a_{l}, a_{h}\right]} u(x, a)$ is strictly increasing continuous and $\pi(x)$ is strictly decreasing continuous within $[c, h], \pi(x)$ and $\max _{a \in\left[a_{l}, a_{h}\right]} u(x, a)$ have a unique intersection within $(c, h)$. Denote 
the horizontal coordinate of the intersection as $x_{0}$. Since $\max _{a \in\left[a_{l}, a_{h}\right]} u(x, a)$ is strictly increasing within $[l, h], \forall x \in\left[l, x_{0}\right)$, we have

$$
\min \left(\pi(x), \max _{a \in\left[a_{l}, a_{h}\right]} u(x, a)\right) \leq \max _{a \in\left[a_{l}, a_{h}\right]} u(x, a)<\max _{a \in\left[a_{l}, a_{h}\right]} u\left(x_{0}, a\right) .
$$

Meanwhile, $\forall x \in\left(x_{0}, h\right]$, we know

$$
\min \left(\pi(x), \max _{a \in\left[a_{l}, a_{h}\right]} u(x, a)\right) \leq \pi(x)<\pi\left(x_{0}\right) .
$$

Since $\quad \pi\left(x_{0}\right)=\max _{a \in\left[a_{l}, a_{h}\right]} u\left(x_{0}, a\right) \quad, \quad x=x_{0} \quad$ satisfies $\quad \max _{x \in[l, h]} \min \left(\pi(x), \max _{a \in\left[a_{l}, a_{h}\right]} u(x, a)\right) \quad$. Considering Lemma 11(1), we know $x_{1}^{*}$ satisfies $\pi(x)=\max _{a \in\left[a_{l}, a_{h}\right]} u(x, a), \quad x \in(c, h)$ and $a_{1}^{*}=\underset{a \in\left[a_{l}, a_{h}\right]}{\arg \max } u\left(x_{1}^{*}, a\right)$.

(2) Since $\max _{a \in\left[a_{l}, a_{h}\right]} u(x, a)$ is strictly increasing within $[l, h], \forall x \neq h$ we have $\max _{a \in\left[a_{l}, a_{h}\right]} u(x, a)<\max _{a \in\left[a_{l}, a_{h}\right]} u(h, a)=1$. Meanwhile, $1-\pi(h)=1$ holds. Considering Lemma 11(2), we know $x_{4}^{*}=h$ and $a_{4}^{*}=\underset{a \in\left[a_{l}, a_{h}\right]}{\arg \max } u(h, a)$.

Likewise, we can prove Theorem 13(3) and 13(4).

\section{Newsvendor models with the one-shot decision theory}

Consider a retailer who sells an innovative product. The retailer orders $q$ units before the season at the unit wholesale price $W$. When the demand $x$ is observed, the retailer sells units (limited by the supply $q$ and the demand $x$ ) at the exogenous unit revenue $R$ with $R>W$. Any excess product can be salvaged at the unit salvage price $S_{o}>0$ with $W>S_{o}$. If there is a shortage, the unit opportunity cost is $S_{u}>0$. The profit function of the retailer is as follows:

$$
r(x, q)=\left\{\begin{array}{ll}
R x+(q-x) S_{o}-W q ; & x<q \\
(R-W) q-S_{u}(x-q) ; & x \geq q
\end{array} .\right.
$$

The plausible information of the demand $x$ is represented by a possibility distribution $\pi_{D}(x)$, that is,

$$
\pi_{D}:\left[d_{l}, d_{u}\right] \rightarrow[0,1] .
$$


$\pi_{D}(x)$ satisfies $\exists d_{c} \in\left(d_{l}, d_{u}\right), \pi_{D}\left(d_{c}\right)=1, \quad \pi_{D}\left(d_{l}\right)=0$ and $\pi_{D}\left(d_{u}\right)=0 . \quad \pi_{D}(x)$ is

strictly quasi-concave continuous; $d_{l}$ and $d_{u}$ are the lower and upper bounds of the demand,

respectively; $d_{c}$ is the most possible amount of the demand. The smaller the possibility degree of the demand $x$ is, the more surprising the occurrence of $x$ is. It should be noted that $\pi_{D}\left(d_{l}\right)=0$ and $\pi_{D}\left(d_{u}\right)=0$ mean that if $d_{l}$ or $d_{u}$ happens it will be most surprising.

However, they do not mean that $d_{l}$ and $d_{u}$ are impossible. The approach for identifying the possibility distribution of the innovative product demand will be given in Section 4 .

Because the demand is inside the interval $\left[d_{l}, d_{u}\right]$, a reasonable order quantity should also lie in this region. The highest profit of retailer is

$$
r_{u}=(R-W) d_{u}
$$

that is, the retailer orders the most $q=d_{u}$ and the demand is the largest $d_{u}$. The lowest profit is $r_{l}=\min \left\{d_{l} R+\left(d_{u}-d_{l}\right) S_{o}-d_{u} W, d_{l} R-\left(d_{u}-d_{l}\right) S_{u}-d_{l} W\right\}$. It is determined by the minimum of two cases: in the first case, the retailer orders the most but the demand is the lowest: $d_{l} R+\left(d_{u}-d_{l}\right) S_{o}-d_{u} W$; in the second case, the retailer orders the lowest but the demand is the highest: $d_{l} R-\left(d_{u}-d_{l}\right) S_{u}-d_{l} W$. We assume $W \geq S_{o}+S_{u}$, which leads to

$$
r_{l}=d_{l} R+\left(d_{u}-d_{l}\right) S_{o}-d_{u} W
$$

The satisfaction function of the retailer is the following strictly increasing function of the profit $r$,

$$
u:\left[r_{l}, r_{u}\right] \rightarrow[0,1]
$$

where $u\left(r_{l}\right)=0$ and $u\left(r_{u}\right)=1$.

(46) is a general form of the satisfaction function of the retailer where the satisfaction degree of the lowest profit is 0 and the satisfaction degree of the highest profit is 1 . The satisfaction 
function is written as $u(r(x, q))$ in the following parts.

\section{Proposition 14.}

(1) $u(r(x, q))$ is strictly increasing continuous in $x$ when $q \geq x$ and strictly decreasing continuous in $x$ when $q \leq x$.

(2) $u(r(x, q))$ is strictly increasing continuous in $q$ when $x \geq q$ and strictly decreasing continuous in $q$ when $x \leq q$.

(3) $\max _{q} u(r(x, q))=u(r(x, x))$ is strictly increasing continuous.

Since the life cycle of the innovative product is generally shorter than the procurement lead-time, the retailer has only one chance to determine the order quantity and one and only demand will occur. It is reasonable that the retailer needs to contemplate which demand ought to be taken into account before ordering products. The retailer chooses one demand (focus point) amongst all possible ones while considering the possibility degree to which the demand will appear in the future and the satisfaction level that the demand can bring about for an order quantity. We consider four types of focus points introduced in Section 2 as follows:

Active focus point of an order quantity: The active focus point of an order quantity $q$, denoted as $x_{1}^{*}(q)$, is

$$
x_{1}^{*}(q)=\underset{x \in\left[d_{l}, d_{u}\right]}{\arg \max } \min \left[\pi_{D}(x), u(r(x, q))\right] .
$$

$x_{1}^{*}(q)$ is a demand that has a higher possibility degree and a higher satisfaction level for an order quantity $q$.

Passive focus point of an order quantity: The passive focus point of an order quantity $q$, denoted as $x_{2}^{*}(q)$, is

$$
x_{2}^{*}(q)=\underset{x \in\left[d_{l}, d_{u}\right]}{\arg \min } \max \left[1-\pi_{D}(x), u(r(x, q))\right] .
$$

$x_{2}^{*}(q)$ is a demand that has a higher possibility degree and a lower satisfaction level for an order quantity $q$.

Apprehensive focus point of an order quantity: The apprehensive focus point of an order quantity $q$, denoted as $x_{3}^{*}(q)$, is

$$
x_{3}^{*}(q)=\underset{x \in\left[d_{l}, d_{u}\right]}{\arg \min } \max [\pi(x), u(r(x, q))] .
$$


$x_{3}^{*}(q)$ is a demand with a lower possibility degree and a lower satisfaction level for an order quantity $q$.

Daring focus point of an order quantity: The daring focus point of an order quantity $q$, denoted as $x_{4}^{*}(q)$, is

$$
x_{4}^{*}(q)=\underset{x \in\left[d_{l}, d_{u}\right]}{\arg \min } \max [\pi(x), 1-u(r(x, q))] .
$$

$x_{4}^{*}(q)$ is a demand with a lower possibility degree and a higher satisfaction level for an order quantity $q$.

For one order quantity, more than one demand might exist as one type of focus point. We denote the sets of four types of focus points of an order quantity $q$ as $X_{1}(q), X_{2}(q), X_{3}(q)$ and $X_{4}(q)$, respectively.

In the newsvendor problem, the retailer contemplates that the focus point is the most appropriate scenarios (demand) for each order quantity and chooses one order quantity which can bring about the best consequence (highest satisfaction level) with the assumption that only focus points come true. The optimal order quantities are obtained as follows:

$$
\begin{aligned}
& q_{1}^{*}=\underset{q \in\left[d_{l}, d_{u}\right]}{\arg \max } \max _{x_{1}^{*}(q) \in X_{1}(q)} u\left(r\left(x_{1}^{*}(q), q\right)\right), \\
& q_{2}^{*}=\underset{q \in\left[d_{l}, d_{u}\right]}{\arg \max } \min _{x_{2}^{*}(q) \in X_{2}(q)} u\left(r\left(x_{2}^{*}(q), q\right)\right), \\
& q_{3}^{*}=\underset{q \in\left[d_{l}, d_{u}\right]}{\arg \max } \min _{x_{3}^{*}(q) \in X_{3}(q)} u\left(r\left(x_{3}^{*}(q), q\right)\right), \\
& q_{4}^{*}=\underset{q \in\left[d_{l}, d_{u}\right]}{\arg \max } \max _{x_{4}^{*}(q) \in X_{4}(q)} u\left(r\left(x_{4}^{*}(q), q\right)\right) .
\end{aligned}
$$

$q_{1}^{*}, q_{2}^{*}, q_{3}^{*}$ and $q_{4}^{*}$ are called optimal active, passive, apprehensive and daring order quantities, respectively. $x_{1}^{*}\left(q_{1}^{*}\right), x_{2}^{*}\left(q_{2}^{*}\right), x_{3}^{*}\left(q_{3}^{*}\right)$ and $x_{4}^{*}\left(q_{4}^{*}\right)$ are optimal active, passive, apprehensive and daring demands, respectively. The retailer who takes into account the active, passive, apprehensive or daring focus point is called active, passive, apprehensive or daring retailer, respectively. 
Comments: The differences between the newsvendor model with the subjective expected utility theory (Newsvendor-SEU) and the newsvendor model with the one-shot decision theory (Newsvendor-OSDT) is shown below.

1. In Newsvendor-SEU, there are two steps as follows:

Step 1: Evaluating each order quantity by the weighted average of the utilities of all payoffs brought about by all possible demands;

Step2: Selecting the order quantity with the maximum average.

In Newsvendor-OSDT, there are two steps as follows:

Step 1: Seeking an appropriate demand (focus point) for each order quantity;

Step 2: Choosing the order quantity with the maximum satisfaction level of the focus point (selected demand).

2. In Newsvendor-SEU, a utility function is associated with risky situations. If a person is a risk averter, the utility function is concave; if a person is a risk taker, the utility function is convex; if a person is risk neutral, the utility function is linear. In Newsvendor-OSDT, the satisfaction function has no relationship with risk situations. It represents the relative position of the payoff. Which type of focus point is used for making a decision reflects the attitude of a decision maker about uncertainty.

3. Newsvendor-SEU uses the subjective probability to characterize uncertainty whereas Newsvendor-OSDT utilizes the possibility distribution.

4. Newsvendor-SEU and Newsvendor-OSDT explain why some order quantity is optimal in different ways. In Newsvendor-SEU an order quantity is evaluated based on the average; that is, if the optimal order quantity is chosen every time then the total utility almost surely attains the maximum in the sense of the strong law of large numbers. However, Newsvendor-OSDT gives a clear answer to why some order quantity is optimal when only one decision chance is left to a retailer.

Let us further clarify the relationship between Newsvendor-OSDT and other existing newsvendor models with probabilities. Newsvendor-OSDT is mainly utilized in the situation where the decision for an order quantity is experienced only once and the probability distribution is unavailable due to lack of enough information. However, it can play an indispensable role of a bridge in linking decision under ignorance and decision with probabilities (shown in Fig. 2). For the first time to sell a new product, a retailer has to make an order under ignorance because the decision situation is completely new for him/her and therefore he/she has no ability to tell the difference between each possible demand. After the first order is made based on maximin or maximax or minmax regret or Hurwicz criterion, he/she has some knowledge about demand so that it is possible to construct an initial possibility distribution of demand. He/she can make a one-shot decision and repeat such a one-shot 
decision with the updated possibility distributions. As time progresses, the information improves. The possibility distribution will switch into a probability distribution when the data is rich enough. The switching criterion is the hypothesis test for the probability distribution. After that newsvendor models based on probability distributions are utilized. Hence, we can say Newsvendor-OSDT is the necessary complement to the newsvendor literature.

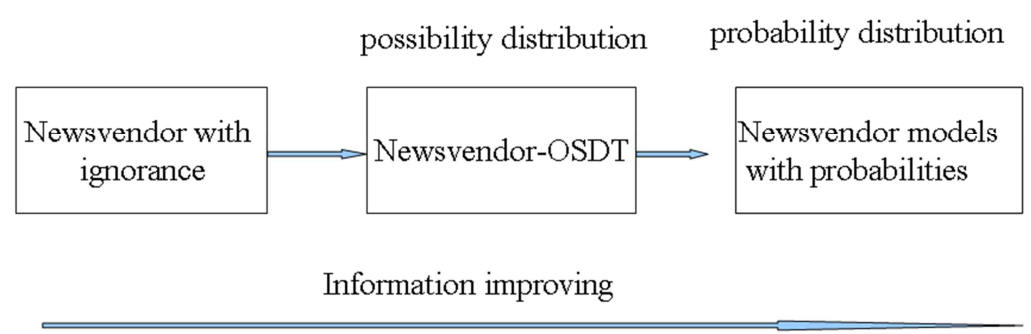

Time progressing

Fig. 2. The relationship amongst three kinds of newsvendor models

Following the idea of Fisher (1997), the innovative products are featured by the unpredictable demand. In such situations, without the use of statistical methods, judgmental forecasting methods; such as, jury of executive opinion and Delphi method are widely used in practice. Such methods take advantage of experts' judgment. In the following section, we propose an approach for obtaining the possibility distribution of the demand. The obtained possibility distribution reflects the expert's knowledge on the demand of the innovative product.

\section{Approaches for identifying the possibility distribution of the innovative product demand}

Consider a retailer who sells an innovative product where no historical data is available for statistical demand analysis. An approach for identifying the possibility distribution of the demand of an innovative product is proposed as follows:

Step 1: Consider an initial reference range of the possible demand, i.e. $[L, H]$. Divide $[L, H]$ into $n$ subsections and denote them as $S_{1}, S_{2}, \cdots, S_{n}$ from left to right. The number and lengths of subsections depend on the knowledge of an expert. The lengths of these subsections are not required to be equal.

Step 2: Ask an expert to make a comparison between each pair of subsections about where the demand is expected to fall. The answer is denoted as $a_{i j}$ based on the following meaning:

1) $a_{i j}=1: S_{i}$ and $S_{j}$ are equally likely to contain the demand;

2) $a_{i j}=3: S_{i}$ is fairly likely to contain the demand than $S_{j}$;

3) $a_{i j}=5: S_{i}$ is a little more likely to contain the demand than $S_{j}$; 
4) $a_{i j}=7: S_{i}$ is much more likely to contain the demand than $S_{j}$;

5) $a_{i j}=9: S_{i}$ is most likely to contain the demand than $S_{j}$;

and the other numbers 2, 4, 6 and 8 are used for the supplementary. It is reasonable to assume that $a_{i j}=1 / a_{j i}$. Then we obtain a comparison matrix as follows:

$$
A=\left[\begin{array}{cccc}
1 & a_{12} & \cdots & a_{1 n} \\
1 / a_{12} & 1 & \cdots & a_{2 n} \\
\vdots & \vdots & \cdots & \vdots \\
1 / a_{1 n} & 1 / a_{2 n} & \cdots & 1
\end{array}\right]
$$

Step 3: Obtain the eigenvector $\vec{p}_{o}{ }^{t}=\left[v_{o 1}, v_{o 2}, \cdots, v_{o n}\right]$ of the matrix $A$ with the largest eigenvalue $\lambda_{\max }$, and normalize it as follows:

$$
\vec{p}^{t}=\vec{p}_{o}^{t} / \max \left(v_{o 1}, v_{o 2}, \cdots, v_{o n}\right)=\vec{p}_{o}^{t} / v_{o k}=\left[v_{1}, v_{2}, \cdots, v_{n}\right]
$$

where the $k$ th entry of $\vec{p}_{o}$ is assumed to be maximum.

Step 4: Choose the middle point of each subsection as its representative. Assume that the coordinate of the representative of the subsection $S_{i}$ is $m_{i}$. It is reasonable to set the possibility degree of $m_{i}$ as $v_{i}$. Denote the possibility distribution with the parameter vector $\boldsymbol{\theta}$ as $\pi(x, \boldsymbol{\theta})$. The following optimization problem is used to seek the parameter vector $\boldsymbol{\theta}$ to make $\pi\left(x_{i}, \boldsymbol{\theta}\right)$ approach to $v_{i}$ from the upper direction as much as possible.

$$
\begin{aligned}
& \min _{\boldsymbol{\theta}} \sum_{i} \pi\left(x_{i}, \boldsymbol{\theta}\right) \\
& \text { s.t. } \pi\left(x_{i}, \boldsymbol{\theta}\right) \geq v_{i}, \quad i=1, \cdots, n .
\end{aligned}
$$

Suppose $\pi(x, \boldsymbol{\theta})$ is a triangular function, denoted as $\left(x_{c}, c_{l}, c_{r}\right)_{t}$, i.e.

$$
\pi(x, \boldsymbol{\theta})=\left(x_{c}, c_{l}, c_{r}\right)_{t}=\left\{\begin{array}{cc}
0, & x<x_{c}-c_{l}, \\
1-\left(x_{c}-x\right) / c_{l}, & x_{c}-c_{l} \leq x \leq x_{c} \\
1-\left(x-x_{c}\right) / c_{r}, & x_{c} \leq x \leq x_{c}+c_{r} \\
0, & x>x_{c}+c_{r}
\end{array},\right.
$$

where the parameter vector $\boldsymbol{\theta}$ is $\left[x_{c}, c_{l}, c_{r}\right]^{t}$, which is needed to be identified. $x_{c}, c_{l}$ and 
$c_{r}$ are the center, the left and right spreads, respectively. Obviously, $x_{c}=m_{k}$. From (57), we obtain

$$
\begin{aligned}
& c_{l}=\max _{i=1, \cdots, k-1} \frac{m_{k}-m_{i}}{1-v_{i}}, \\
& c_{r}=\max _{i=k+1, \cdots, n} \frac{m_{i}-m_{k}}{1-v_{i}} .
\end{aligned}
$$

The range $\left[L^{\prime}, H^{\prime}\right]$ is called the revised range of the possible demand where $L^{\prime}=m_{k}-c_{l}$ and $H^{\prime}=m_{k}+c_{r}$.

In the identification approach, the pairwise comparison is used for obtaining the possibility degrees of some discrete values of the demand. In fact, pairwise comparisons are commonly used in the analytic hierarchy process (AHP) (Saaty, 1980) for multiple criteria decision making (MCDM) problems with the common knowledge that eliciting indirect preference is less demanding of cognitive effort. In AHP, $a_{i j}$ represents the degree to which the criteria $i$ is

more important than the criteria $j$; and in this research, $a_{i j}$ represents the degree to which the subsection $i$ is more possible to contain the demand than the subsection $i$. In AHP, the eigenvector with the largest eigenvalue is used to reflect the weights of decision criteria. Using the similar idea, the normalized eigenvector with the largest eigenvalue is used to present the possibility degrees to which the demand is contained by the subsections. Recently, Guo and Tanaka (2010) proposed a method for estimating interval probabilities based on pairwise comparisons of the likelihoods of events. The point-valued pairwise comparison is included by the ratio of the estimated interval probabilities. The interval probabilities are obtained by linear programming problems. As its extension, Guo and Wang (2012) considered the interval-valued comparison and proposed an approach to identify the dual interval probabilities.

\section{Analysis results of OSDT based newsvendor models}

In this section the plausible demand information is characterized by the possibility distribution $\pi_{D}(x)$ shown in (43) and identified by the proposed method in Section 4. Let us think about how to obtain the optimal active, passive, apprehensive and daring order quantities.

Lemma 15. The optimal active order quantity $q_{1}^{*}$ is the solution of the following equation:

$$
u(r(x, x))=\pi_{D}(x), x \in\left(d_{c}, d_{u}\right)
$$

The optimal active demand, i.e. $x_{1}^{*}\left(q_{1}^{*}\right)$ is $q_{1}^{*}$. 
Proof. The proof follows directly from Theorem 13 (1) and Proposition 14(3).

Interestingly, Lemma 15 indicates that the focus point (selected demand) of the active retailer's optimal order quantity is the optimal order quantity itself. It means that the active retailer has confidence that he/she can sell all the products that he/she has optimally ordered.

Lemma 16. The optimal passive order quantity $q_{2}^{*}$ is the solution of the following equation:

$$
u\left(r\left(d_{p l}(q), q\right)\right)=u\left(r\left(d_{p u}(q), q\right)\right)
$$

equivalently,

$$
\pi_{D}\left(d_{p l}(q)\right)=\pi_{D}\left(d_{p u}(q)\right)
$$

where $d_{p l}(q)$ and $d_{p u}(q)$ are the horizontal coordinates of the intersections of $u(r(x, q))$ and $1-\pi_{D}(x)$ within $\left[d_{l}, \min \left(q, d_{c}\right)\right]$ and $\left[\max \left(q, d_{c}\right), d_{u}\right]$, respectively. The optimal passive demand, i.e. $x_{2}^{*}\left(q_{2}^{*}\right)$ are $d_{p l}\left(q_{2}^{*}\right)$ and $d_{p u}\left(q_{2}^{*}\right)$.

Proof. It follows from Proposition 14(3) that $u(r(x, x))$ is strictly increasing continuous within $\left[d_{l}, d_{u}\right] .1-\pi_{D}(x)$ is strictly decreasing continuous within $\left[d_{l}, d_{c}\right]$ and strictly increasing continuous within $\left[d_{c}, d_{u}\right]$. Since $u\left(r\left(d_{l}, d_{l}\right)\right)<1-\pi_{D}\left(d_{l}\right)=1 \quad$ and $u\left(r\left(d_{c}, d_{c}\right)\right)>1-\pi_{D}\left(d_{c}\right)=0$ and $u\left(r\left(d_{u}, d_{u}\right)\right)=1-\pi_{D}\left(d_{u}\right)=1$, there is a unique intersection of $u(r(x, x))$ and $1-\pi_{D}(x)$ within $\left(d_{l}, d_{c}\right)$, the horizontal coordinate of this intersection is denoted as $d_{p l}^{*}$; and there is at least one intersection within $\left(d_{c}, d_{u}\right]$, the minimum horizontal coordinate of these intersections is denoted as $d_{p u}^{*}$. In what follows, we consider three cases of $q$, i.e. $q \in\left[d_{l}, d_{p l}^{*}\right], q \in\left[d_{p u}^{*}, d_{u}\right]$ and $q \in\left[d_{p l}^{*}, d_{p u}^{*}\right]$.

Case 1: $q \in\left[d_{l}, d_{p l}^{*}\right]$. That is, $u(r(q, q)) \leq 1-\pi_{D}(q)$ and $q<d_{c}$. It follows from Theorem 2(3) that the passive focus point of $q \in\left[d_{l}, d_{p l}^{*}\right]$ is $x_{2}^{*}(q)=d_{p u}(q)$. From Proposition $14(2)$ and Corollary 5(2), we have 


$$
\max _{q \in\left[d_{l}, d_{p l}^{*}\right]} u\left(r\left(x_{2}(q), q\right)\right)=\max _{q \in\left[d_{l}, d_{p l}^{*}\right]} u\left(r\left(d_{p u}(q), q\right)\right)=u\left(r\left(d_{p u}\left(d_{p l}^{*}\right), d_{p l}^{*}\right)\right)
$$

Case 2: $\quad q \in\left[d_{p u}^{*}, d_{u}\right]$. For the case $u(r(q, q)) \leq 1-\pi_{D}(q)$, Theorem 2(2) shows that the passive focus point is $x_{2}(q)=d_{p l}(q)$. In the case $u(r(q, q)) \geq 1-\pi_{D}(q)$, since $u\left(r\left(d_{p u}^{*}, q\right)\right) \leq u\left(r\left(d_{p u}^{*}, d_{p u}^{*}\right)\right)=1-\pi_{D}\left(d_{p u}^{*}\right) \quad$ and $\quad F(x)=u(r(x, q))-\left(1-\pi_{D}(x)\right) \quad$ is $\quad$ a continuous function, $\exists x_{0} \in\left[d_{p u}^{*}, q\right] \quad F\left(x_{0}\right)=u\left(r\left(x_{0}, q\right)\right)-\left(1-\pi_{D}\left(x_{0}\right)\right)=0$ holds. From Corollary 3(1), we know that the passive focus point is $x_{2}(q)=d_{p l}(q)$. Therefore, $\forall q \in\left[d_{p u}^{*}, d_{u}\right]$, the passive focus point is $x_{2}(q)=d_{p l}(q)$. From Proposition 14(2) and Corollary 5(1), we have

$$
\max _{q \in\left[d_{p u}^{*}, d_{u}\right]} u\left(r\left(x_{2}(q), q\right)\right)=u\left(r\left(d_{p l}(q), q\right)\right)=u\left(r\left(d_{p l}\left(d_{p u}^{*}\right), d_{p u}^{*}\right)\right) .
$$

Case 3: $q \in\left[d_{p l}^{*}, d_{p u}^{*}\right]$. That is, $u(r(q, q)) \geq 1-\pi_{D}(q)$. It follows from Theorem 2(1) that

$$
u\left(r\left(x_{2}(q), q\right)\right)=\min \left(u\left(r\left(d_{p l}(q), q\right)\right), u\left(r\left(d_{p u}(q), q\right)\right)\right)
$$

From Theorem 8 , we know that $u\left(r\left(d_{p l}(q), q\right)\right)$ and $u\left(r\left(d_{p u}(q), q\right)\right)$ are uniformly continuous in $q \in\left[d_{p l}^{*}, d_{p u}^{*}\right]$. Considering $F(q)=u\left(r\left(d_{p l}(q), q\right)\right)-u\left(r\left(d_{p u}(q), q\right)\right)$, it follows from Proposition 14(2) and Corollary 5 that $F(q)$ is strictly decreasing within $\left[d_{p l}^{*}, d_{p u}^{*}\right]$. For $q=d_{p l}^{*}$ and $q=d_{p u}^{*}$, we have

$$
\begin{aligned}
& F\left(d_{p l}^{*}\right)=u\left(r\left(d_{p l}\left(d_{p l}^{*}\right), d_{p l}^{*}\right)\right)-u\left(r\left(d_{p u}\left(d_{p l}^{*}\right), d_{p l}^{*}\right)\right)>0 \\
& F\left(d_{p u}^{*}\right)=u\left(r\left(d_{p l}\left(d_{p u}^{*}\right), d_{p u}^{*}\right)\right)-u\left(r\left(d_{p u}\left(d_{p u}^{*}\right), d_{p u}^{*}\right)\right)<0 .
\end{aligned}
$$

Therefore, there is a unique $\widetilde{q} \in\left(d_{p l}^{*}, d_{p u}^{*}\right)$ satisfying $u\left(r\left(d_{p l}(\widetilde{q}), \widetilde{q}\right)\right)=u\left(r\left(d_{p u}(\widetilde{q}), \widetilde{q}\right)\right)$. Theorem 2(1) shows 


$$
x_{2}(\widetilde{q})=\left\{d_{p l}(\widetilde{q}), d_{p u}(\widetilde{q})\right\}
$$

Let us consider the case $q>\widetilde{q}$. From Proposition $14(2)$, we know $u(r(x, q))<u(r(x, \widetilde{q}))$ for $x \in\left[d_{l}, \widetilde{q}\right]$; and $u(r(x, q))>u(r(x, \widetilde{q}))$ for $x \in\left[q, d_{u}\right]$. From Corollary 5, we have

$$
\begin{aligned}
& u\left(r\left(d_{p l}(q), q\right)\right)<u\left(r\left(d_{p l}(\widetilde{q}), \widetilde{q}\right)\right), \\
& u\left(r\left(d_{p u}(q), q\right)\right)>u\left(r\left(d_{p u}(\widetilde{q}), \widetilde{q}\right)\right) .
\end{aligned}
$$

From Theorem 2(1), we have

$$
\max _{q \in\left[\widetilde{q}, d_{p u}^{*}\right]} u\left(r\left(x_{2}(q), q\right)\right)=u\left(r\left(x_{2}(\widetilde{q}), \widetilde{q}\right)\right) .
$$

Likewise, we have

$$
\max _{q \in\left[d_{p l}^{*}, \widetilde{q}\right]} u\left(r\left(x_{2}(q), q\right)\right)=u\left(r\left(x_{2}(\widetilde{q}), \widetilde{q}\right)\right) .
$$

From (64), (65), (72) and (73), we know $q_{2}^{*}=\underset{q \in\left[d_{l}, d_{u}\right]}{\arg \max } u\left(r\left(x_{2}(q), q\right)\right)=\widetilde{q}$, which means that (62) and (63) hold. (69) means that the optimal passive demand, i.e. $x_{2}^{*}\left(q_{2}^{*}\right)$ are $d_{p l}\left(q_{2}^{*}\right)$ and $d_{p u}\left(q_{2}^{*}\right)$

Lemma 16 implies that the passive retailer chooses the optimal order quantity which makes its two focus points (selected demands) have the same possibility degrees and the same satisfaction levels.

Lemma 17. The optimal apprehensive order quantity $q_{3}^{*}$ is the solution of

$$
u\left(r\left(d_{l}, q\right)\right)=u\left(r\left(d_{u}, q\right)\right)
$$

that is,

$$
q_{3}^{*}=\frac{\left(R-S_{o}\right) d_{l}+S_{u} d_{u}}{R-S_{o}+S_{u}} .
$$

The optimal apprehensive demand, i.e. $x_{3}^{*}\left(q_{3}^{*}\right)$ are $d_{l}$ and $d_{u}$.

Proof: It follows from Lemma 6 that $\forall q \in\left[d_{l}, d_{u}\right]$ we have

$$
u\left(r\left(x_{3}(q), q\right)\right)=\min \left(u\left(r\left(d_{l}, q\right)\right), u\left(r\left(d_{u}, q\right)\right)\right),
$$

which leads to

$$
q_{3}^{*}=\underset{q \in\left[d_{l}, d_{u}\right]}{\arg \max } u\left(r\left(x_{3}(q), q\right)\right)=\underset{q \in\left[d_{l}, d_{u}\right]}{\arg \max } \min \left(u\left(r\left(d_{l}, q\right)\right), u\left(r\left(d_{u}, q\right)\right)\right) .
$$


Suppose $\widetilde{q}$ is the solution of $u\left(r\left(d_{l}, q\right)\right)=u\left(r\left(d_{u}, q\right)\right)$, that is,

$$
u\left(r\left(d_{l}, \widetilde{q}\right)\right)=u\left(r\left(d_{u}, \widetilde{q}\right)\right)
$$

Proposition 14(2) shows if $q<\widetilde{q}$ then $u\left(r\left(d_{l}, q\right)\right)>u\left(r\left(d_{u}, q\right)\right)$ and if $q>\widetilde{q}$ then $u\left(r\left(d_{l}, q\right)\right)<u\left(r\left(d_{u}, q\right)\right)$ so that we have

$$
\begin{aligned}
& \max _{q \in\left[d_{l}, d_{u}\right]} \min \left(u\left(r\left(d_{l}, q\right)\right), u\left(r\left(d_{u}, q\right)\right)\right) \\
& =\max _{q \in\left[d_{l}, \widetilde{q}\right]} \min \left(u\left(r\left(d_{l}, q\right)\right), u\left(r\left(d_{u}, q\right)\right)\right) \vee \max _{q \in\left[\widetilde{q}, d_{u}\right]} \min \left(u\left(r\left(d_{l}, q\right)\right), u\left(r\left(d_{u}, q\right)\right)\right) \\
& =\max _{q \in\left[d_{l}, \widetilde{q}\right]} u\left(r\left(d_{u}, q\right)\right) \vee \max _{q \in\left[\widetilde{q}, d_{u}\right]} u\left(r\left(d_{l}, q\right)\right)=u\left(r\left(d_{u}, \widetilde{q}\right)\right) \vee u\left(r\left(d_{l}, \widetilde{q}\right)\right) .
\end{aligned}
$$

Therefore, $q_{3}^{*}$ is $\widetilde{q}$, which is the solution of (74). (74) leads to (75) with considering (42).

(23) implies that $x_{3}^{*}\left(q_{3}^{*}\right)$ are $d_{l}$ and $d_{u}$.

Lemma 17 shows that the apprehensive retailer takes into account two extreme demands (the highest and the lowest demand) and chooses the optimal order quantity which makes the satisfaction levels of the highest demand and the lowest demand equal.

Lemma 18. The optimal daring order quantity is

$$
q_{4}^{*}=d_{u}
$$

The optimal daring demand, i.e. $x_{4}^{*}\left(q_{4}^{*}\right)$ is $d_{u}$.

Proof. The proof follows directly from Theorem 13(2) and Proposition 14(3).

According to Lemma 18, for the daring retailer, the highest demand is his/her optimal order quantity and he/she believes all ordered products can be sold.

It is helpful to discuss the relationships amongst the four types of optimal order quantities and focus points and how the four types of optimal order quantities and focus points change with the parameters. We have the following lemmas.

\section{Lemma 19.}

(1) The optimal daring order quantity $q_{4}^{*}$ is always larger than any other type of optimal order quantity. 
(2) Supposing the possibility distribution $\pi_{D}(x)$ is symmetric, we have

$$
\begin{aligned}
& d_{p l}\left(q_{2}^{*}\right)+d_{p u}\left(q_{2}^{*}\right)=d_{l}+d_{u}<2 x_{1}\left(q_{1}^{*}\right)<2 d_{u}, \\
& q_{1}^{*}>q_{2}^{*}>q_{3}^{*} .
\end{aligned}
$$

\section{Proof.}

(1) It is straightforward that $q_{4}^{*}$ is always larger than any other type of optimal order quantity.

(2) From Lemma 16 and the monotonicity of $u(r(x, q))$, we have

$$
r\left(d_{p l}\left(q_{2}^{*}\right), q_{2}^{*}\right)=r\left(d_{p u}\left(q_{2}^{*}\right), q_{2}^{*}\right),
$$

which is equal to

$$
R d_{p l}\left(q_{2}^{*}\right)+S_{o}\left(q_{2}^{*}-d_{p l}\left(q_{2}^{*}\right)\right)-W q_{2}^{*}=(R-W) q_{2}^{*}-S_{u}\left(d_{p u}\left(q_{2}^{*}\right)-q_{2}^{*}\right) .
$$

From (84), we obtain

$$
q_{2}^{*}=\frac{\left(R-S_{o}\right) d_{p l}\left(q_{2}^{*}\right)+S_{u} d_{p u}\left(q_{2}^{*}\right)}{\left(R-S_{o}\right)+S_{u}} .
$$

Hence,

$$
q_{1}^{*}-q_{2}^{*}=d_{o u}^{*}-\frac{\left(R-S_{o}\right) d_{p l}\left(q_{2}^{*}\right)+S_{u} d_{p u}\left(q_{2}^{*}\right)}{\left(R-S_{o}\right)+S_{u}}=\frac{\left(R-S_{o}\right)\left(d_{o u}^{*}-d_{p l}\left(q_{2}^{*}\right)\right)-S_{u}\left(d_{p u}\left(q_{2}^{*}\right)-d_{o u}^{*}\right)}{\left(R-S_{o}\right)+S_{u}},
$$

where $q_{1}^{*}=d_{o u}^{*}$ is the horizontal coordinate of the intersection of $u(r(x, x))$ and $\pi_{D}(x)$ within $\left(d_{c}, d_{u}\right)$ (See Lemma 15). From (63), we have

$$
\pi\left(d_{p l}\left(q_{2}^{*}\right)\right)=\pi\left(d_{p u}\left(q_{2}^{*}\right)\right)
$$

If $\pi_{D}(x)$ is symmetric, (87) implies

$$
d_{p l}\left(q_{2}^{*}\right)-d_{l}=d_{u}-d_{p u}\left(q_{2}^{*}\right)>0 \text {. }
$$

(88) is equal to

$$
d_{p l}\left(q_{2}^{*}\right)+d_{p u}\left(q_{2}^{*}\right)=d_{l}+d_{u}=2 d_{c}<2 d_{o u}^{*}<2 d_{u},
$$

which proves (81). From (89), we have

$$
d_{o u}^{*}-d_{p l}\left(q_{2}^{*}\right)>d_{p u}\left(q_{2}^{*}\right)-d_{o u}^{*} .
$$


Obviously, $d_{o u}^{*}-d_{p l}\left(q_{2}^{*}\right)>0$ holds. Recalling

$$
R-S_{o}>S_{u}>0,
$$

from (86) we know

$$
q_{1}^{*}-q_{2}^{*}>0 \text {. }
$$

By using (85) and (75), we have

$$
\begin{aligned}
q_{2}^{*}-q_{3}^{*} & =\frac{\left(R-S_{o}\right) d_{p l}\left(q_{2}^{*}\right)+S_{u} d_{p u}\left(q_{2}^{*}\right)}{\left(R-S_{o}\right)+S_{u}}-\frac{\left(R-S_{o}\right) d_{l}+S_{u} d_{u}}{\left(R-S_{o}\right)+S_{u}} \\
& =\frac{\left(R-S_{o}\right)\left(d_{p l}\left(q_{2}^{*}\right)-d_{l}\right)-S_{u}\left(d_{u}-d_{p u}\left(q_{2}^{*}\right)\right)}{\left(R-S_{o}\right)+S_{u}} .
\end{aligned}
$$

By using (88) and (91), we know

$$
q_{2}^{*}-q_{3}^{*}>0
$$

(92) and (94) means (82).

Lemma 20. Set the satisfaction function as the following linear function

$$
u(r(x, q))=\frac{r(x, q)-r_{l}}{r_{u}-r_{l}} .
$$

The optimal active order $q_{1}^{*}$ and the optimal active demand $x_{1}^{*}\left(q_{1}^{*}\right)$ are decreasing in the unit wholesale price $W$, increasing in the unit revenue $R$ and the unit salvage price $S_{o}$. The unit opportunity cost $S_{u}$ has no effect on them.

Proof. Since $r_{u}(W)=(R-W) d_{u}$ and $r_{l}(W)=d_{l} R+\left(d_{u}-d_{l}\right) S_{o}-d_{u} W$, by using (95) we have

$$
u(r(W, x, q))=\frac{r(W, x, q)-r_{l}(W)}{r_{u}(W)-r_{l}(W)}=\left\{\begin{array}{cc}
\frac{\left(x-d_{l}\right) R+\left(d_{u}-q\right) W-\left(d_{u}-d_{l}+x-q\right) S_{o}}{\left(d_{u}-d_{l}\right)\left(R-S_{o}\right)} & ; \text { if } x<q \\
\frac{\left(q-d_{l}\right) R+\left(d_{u}-q\right) W-\left(d_{u}-d_{l}\right) S_{o}-(x-q) S_{u}}{\left(d_{u}-d_{l}\right)\left(R-S_{o}\right)} & \text { if } x \geq q
\end{array} .\right.
$$

Therefore,

$$
u(r(W, x, x))=\frac{\left(x-d_{l}\right) R+\left(d_{u}-x\right) W-\left(d_{u}-d_{l}\right) S_{o}}{\left(d_{u}-d_{l}\right)\left(R-S_{o}\right)} .
$$

Differentiating (97) with respect to $W$, we have

$$
\frac{d u(r(W, x, x))}{d W}=\frac{\left(d_{u}-x\right)}{\left(d_{u}-d_{l}\right)\left(R-S_{o}\right)} \geq 0,
$$


which means $\forall W_{1} \leq W_{2} \quad u\left(r\left(W_{1}, x, x\right)\right) \leq u\left(r\left(W_{2}, x, x\right)\right)$ holds so that we have

$$
u\left(r\left(W_{1}, q_{1}^{*}\left(W_{1}\right), q_{1}^{*}\left(W_{1}\right)\right)\right) \leq u\left(r\left(W_{2}, q_{1}^{*}\left(W_{1}\right), q_{1}^{*}\left(W_{1}\right)\right)\right),
$$

where $q_{1}^{*}\left(W_{1}\right)$ is the optimal active order quantity with the wholesale price $W_{1}$. According to Lemma $15, \pi_{D}\left(q_{1}^{*}\left(W_{1}\right)\right)=u\left(r\left(W_{1}, q_{1}^{*}\left(W_{1}\right), q_{1}^{*}\left(W_{1}\right)\right)\right)$ holds so that we have

$$
\pi_{D}\left(q_{1}^{*}\left(W_{1}\right)\right) \leq u\left(r\left(W_{2}, q_{1}^{*}\left(W_{1}\right), q_{1}^{*}\left(W_{1}\right)\right)\right) .
$$

Recalling $u\left(r\left(W_{2}, d_{c}, d_{c}\right)\right)<\pi_{D}\left(d_{c}\right)=1$, due to the monotonicity of $\pi_{D}(x)$ and $u\left(r\left(W_{2}, x, x\right)\right)$ within $\left[d_{c}, q_{1}^{*}\left(W_{1}\right)\right]$, there is one and only one intersection of $\pi_{D}(x)$ and $u\left(r\left(W_{2}, x, x\right)\right)$ within $\left[d_{c}, q_{1}^{*}\left(W_{1}\right)\right]$. Its horizontal coordinate is $q_{1}^{*}\left(W_{2}\right)$ and we have $q_{1}^{*}\left(W_{1}\right) \geq q_{1}^{*}\left(W_{2}\right)$. Therefore, the optimal active order $q_{1}^{*}$ and the active focus point $x_{1}^{*}\left(q_{1}^{*}\right)$ are decreasing in $W$.

Similarly, we can prove the optimal active order $q_{1}^{*}$ and the optimal active demand $x_{1}^{*}\left(q_{1}^{*}\right)$ are increasing in $R$ and $S_{o}$. Since there is not $S_{u}$ in (97), $S_{u}$ has no effect on the optimal active order quantity and the optimal active demand.

Lemma 20 is intuitively obvious if we know that an active retailer believes he/she can sell what he/her optimally orders (shown in Lemma 15).

Let us think about the optimal passive order $q_{2}^{*}$. If the unit opportunity cost price $S_{u}$ increases, $u(r(x, q))$ will remain the same for $x<q$ but decrease for $x \geq q$. Considering Proposition 14(2) and Lemma 16, we have the following proposition.

Proposition 21. The optimal passive order quantity $q_{2}^{*}$ increases in the unit opportunity cost $S_{u}$.

Proposition 21 shows that the passive retailer offsets the loss caused by the increase of the unit opportunity cost by increasing the order quantity.

For the optimal apprehensive order $q_{3}^{*}$, let us consider (75). Obviously, we have

$$
\begin{aligned}
& \frac{d q_{3}^{*}}{d S_{o}}=\frac{\left(d_{u}-d_{l}\right) S_{u}}{\left(R+S_{u}-S_{o}\right)^{2}}>0, \\
& \frac{d q_{3}^{*}}{d S_{u}}=\frac{\left(d_{u}-d_{l}\right)\left(R-S_{o}\right)}{\left(R+S_{u}-S_{o}\right)^{2}}>0,
\end{aligned}
$$




$$
\begin{aligned}
\frac{d q_{3}^{*}}{d R} & =-\frac{\left(d_{u}-d_{l}\right) S_{u}}{\left(R+S_{u}-S_{o}\right)^{2}}<0, \\
\frac{d q_{3}^{*}}{d W} & =0,
\end{aligned}
$$

which can be concluded as the following proposition.

Proposition 22. The optimal apprehensive order quantity $q_{3}^{*}$ increases in the unit salvage price $S_{o}$ and the unit opportunity cost $S_{u}$, decreases in the unit revenue $R$. The unit wholesale price $W$ has no effect on $q_{3}^{*}$.

Proposition 22 points out that for an apprehensive retailer, he/she orders less at the higher unit revenue and the unit wholesale price has no effect on the optimal order quantity. Interestingly, other researchers (Wang and Webster, 2009; Wang et al., 2009) have arrived at similar results in "risk-averse" and "loss-averse" newsvendor models. However, such results were regarded as the limitations of the expected utility theory (EUT) by themselves. Our model can explain these results as follows:

As shown in Lemma 17, the apprehensive retailer worries about two extreme demands, i.e. the smallest demand $d_{l}$ and the largest demand $d_{u}$ and seeks an optimal order quantity to make the satisfaction levels of these two demands equal. (42) shows that for the same order quantity, the increase of the unit revenue will increase the satisfaction level of $d_{u}$ more than $d_{l}$. To offset this effect, the retailer will decrease the order quantity.

For examining the result related to $W$, let us begin with the optimal apprehensive order quantity $q_{3}^{*}$. When the unit wholesale price becomes lower and the order quantity remains the same, the payoff at the demand $d_{u}$ is exactly the same as the one at the demand $d_{l}$. On the other hand, from Proposition 14(2) we know that if the order quantity increases, the satisfaction level of the demand $d_{l}$ will become worse; and if the order quantity decreases, the satisfaction level of the demand $d_{u}$ will become worse. Therefore, the optimal apprehensive order quantity remains $q_{3}^{*}$.

Definition 4. $\pi_{D}^{\prime \prime}(x)$ is said to be more informed than $\pi_{D}^{\prime}(x)$ if and only if $\forall x$ $\pi_{D}^{\prime \prime}(x) \leq \pi_{D}^{\prime}(x)$ holds.

Lemma 23. Suppose $\pi_{D}^{\prime \prime}(x)$ is more informed than $\pi_{D}^{\prime}(x)$. The optimal active order quantities based on possibility distributions $\pi_{D}^{\prime}(x)$ and $\pi_{D}^{\prime \prime}(x)$ are denoted as $q_{o}^{1 *}$ and $q_{o}^{2 *}$, 
respectively; the optimal passive order quantities based on possibility distributions $\pi_{D}^{\prime}(x)$ and $\pi_{D}^{\prime \prime}(x)$ are denoted as $q_{p}^{1 *}$ and $q_{p}^{2 *}$, respectively; the optimal apprehensive order quantities based on possibility distributions $\pi_{D}^{\prime}(x)$ and $\pi_{D}^{\prime \prime}(x)$ are denoted as $q_{a}^{1 *}$ and $q_{a}^{2 *}$, respectively; the optimal daring order quantities based on possibility distributions $\pi_{D}^{\prime}(x)$ and $\pi_{D}^{\prime \prime}(x)$ are denoted as $q_{d}^{1 *}$ and $q_{d}^{2 *}$, respectively. We have

(1) $q_{o}^{1 *} \geq q_{o}^{2 *}$

(2) $q_{a}^{1 *}=q_{a}^{2 *}, q_{d}^{1 *}=q_{d}^{2 *}$

(3) if possibility distributions $\pi_{D}^{\prime}(x)$ and $\pi_{D}^{\prime \prime}(x)$ are symmetric, then $q_{p}^{1 *} \leq q_{p}^{2 *}$.

\section{Proof.}

(1) The horizontal coordinate of the intersection of $u(r(x, x))$ and $\pi_{D}^{\prime}(x)$ within $\left(d_{c}, d_{u}\right)$ is denoted as $d_{o u}^{* 1}$. From Lemma 15 and Definition 4, we know $\pi_{D}^{\prime}\left(d_{o u}^{* 1}\right)=u\left(r\left(d_{o u}^{* 1}, d_{o u}^{* 1}\right)\right) \geq \pi_{D}^{\prime \prime}\left(d_{o u}^{* 1}\right)$. Due to the monotonicity of $\pi_{D}^{\prime \prime}(x)$ and $u(r(x, x))$ and $u\left(r\left(d_{c}, d_{c}\right)\right)<\pi_{D}^{\prime \prime}\left(d_{c}\right)=1$, there is one and only one intersection of $\pi_{D}^{\prime \prime}(x)$ and $u(r(x, x))$ within $\left(d_{c}, d_{o u}^{* 1}\right]$. Its horizontal coordinate is denoted as $d_{o u}^{* 2}$. We have $d_{o u}^{* 1} \geq d_{o u}^{* 2}$, that is, $q_{o}^{1 *} \geq q_{o}^{2 *}$.

(2) It follows directly from Lemmas 17 and 18 that $q_{a}^{1 *}=q_{a}^{2 *}$ and $q_{d}^{1 *}=q_{d}^{2 *}$.

(3) The horizontal coordinates of the intersections of $u(r(x, q))$ and $1-\pi_{D}^{\prime}(x)$ within $\left[d_{l}, \min \left(q, d_{c}\right)\right]$ and $\left[\max \left(q, d_{c}\right), d_{u}\right]$ are denoted as $d_{p l}^{\prime}(q)$ and $d_{p u}^{\prime}(q)$, respectively. The horizontal coordinates of the intersections of $u(r(x, q))$ and $1-\pi_{D}^{\prime \prime}(x)$ within $\left[d_{l}, \min \left(q, d_{c}\right)\right]$ and $\left[\max \left(q, d_{c}\right), d_{u}\right]$ are denoted as $d_{p l}^{\prime \prime}(q)$ and $d_{p u}^{\prime \prime}(q)$, respectively. Lemma 16 shows

$$
u\left(r\left(d_{p l}^{\prime}\left(q_{p}^{1 *}\right), q_{p}^{1 *}\right)\right)=u\left(r\left(d_{p u}^{\prime}\left(q_{p}^{1 *}\right), q_{p}^{1 *}\right)\right),
$$




$$
u\left(r\left(d_{p l}^{\prime \prime}\left(q_{p}^{2 *}\right), q_{p}^{2 *}\right)\right)=u\left(r\left(d_{p u}^{\prime \prime}\left(q_{p}^{2 *}\right), q_{p}^{2 *}\right)\right)
$$

Due to the symmetry of $\pi_{D}^{\prime \prime}(x),(106)$ implies

$$
d_{c}-d_{p l}^{\prime \prime}\left(q_{p}^{2 *}\right)=d_{p u}^{\prime \prime}\left(q_{p}^{2 *}\right)-d_{c} .
$$

From Lemma 16 and Definition 4, we know

$$
\begin{aligned}
& u\left(r\left(q_{p}^{1 *}, q_{p}^{1 *}\right)\right)>1-\pi_{D}^{\prime}\left(q_{p}^{1 *}\right) \\
& u\left(r\left(q_{p}^{2 *}, q_{p}^{2 *}\right)\right)>1-\pi_{D}^{\prime \prime}\left(q_{p}^{2 *}\right) \geq 1-\pi_{D}^{\prime}\left(q_{p}^{2 *}\right) .
\end{aligned}
$$

Suppose $q_{p}^{1 *}>q_{p}^{2 *}$. Considering Proposition 14(2), $u\left(r\left(x, q_{p}^{1 *}\right)\right)<u\left(r\left(x, q_{p}^{2 *}\right)\right)$ holds for any $x \in\left[l, q_{p}^{2 *}\right]$. Considering (108) and using Corollary 5(1), we have

$$
u\left(r\left(d_{p l}^{\prime}\left(q_{p}^{1 *}\right), q_{p}^{1 *}\right)\right)<u\left(r\left(d_{p l}^{\prime}\left(q_{p}^{2 *}\right), q_{p}^{2 *}\right)\right)
$$

Likewise, $u\left(r\left(x, q_{p}^{1 *}\right)\right)>u\left(r\left(x, q_{p}^{2 *}\right)\right)$ holds for any $x \in\left[q_{p}^{1 *}, h\right]$. Considering (109) and using Corollary 5(2), we have

$$
u\left(r\left(d_{p u}^{\prime}\left(q_{p}^{1 *}\right), q_{p}^{1 *}\right)\right)>u\left(r\left(d_{p u}^{\prime}\left(q_{p}^{2 *}\right), q_{p}^{2 *}\right)\right) .
$$

(105), (110) and (111) imply

$$
u\left(r\left(d_{p l}^{\prime}\left(q_{p}^{2 *}\right), q_{p}^{2 *}\right)\right)>u\left(r\left(d_{p u}^{\prime}\left(q_{p}^{2 *}\right), q_{p}^{2 *}\right)\right) .
$$

Due to the symmetry of $\pi_{D}^{\prime}(x),(112)$ means

$$
d_{c}-d_{p l}^{\prime}\left(q_{p}^{2 *}\right)>d_{p u}^{\prime}\left(q_{p}^{2 *}\right)-d_{c}
$$

(107) and (113) mean

$$
d_{p l}^{\prime \prime}\left(q_{p}^{2 *}\right)-d_{p l}^{\prime}\left(q_{p}^{2 *}\right)>d_{p u}^{\prime}\left(q_{p}^{2 *}\right)-d_{p u}^{\prime \prime}\left(q_{p}^{2 *}\right) .
$$

Since $\pi_{D}^{\prime \prime}(x) \leq \pi_{D}^{\prime}(x)$, considering Lemma 16 it is easy to know

$$
d_{p u}^{\prime}\left(q_{p}^{2 *}\right)-d_{p u}^{\prime \prime}\left(q_{p}^{2 *}\right) \geq 0 .
$$

From (42), we have 


$$
\begin{aligned}
& r\left(d_{p l}^{\prime}\left(q_{p}^{2 *}\right), q_{p}^{2 *}\right) \\
& =r\left(d_{p l}^{\prime \prime}\left(q_{p}^{2 *}\right)+\left(d_{p l}^{\prime}\left(q_{p}^{2 *}\right)-d_{p l}^{\prime \prime}\left(q_{p}^{2 *}\right)\right), q_{p}^{2 *}\right) \\
& =r\left(d_{p l}^{\prime \prime}\left(q_{p}^{2 *}\right), q_{p}^{2 *}\right)+\left(R-S_{o}\right)\left(d_{p l}^{\prime}\left(q_{p}^{2 *}\right)-d_{p l}^{\prime \prime}\left(q_{p}^{2 *}\right)\right) .
\end{aligned}
$$

Similarly, we have

$$
\begin{aligned}
& r\left(d_{p u}^{\prime}\left(q_{p}^{2 *}\right), q_{p}^{2 *}\right) \\
& =r\left(d_{p u}^{\prime \prime}\left(q_{p}^{2 *}\right)+\left(d_{p u}^{\prime}\left(q_{p}^{2 *}\right)-d_{p u}^{\prime \prime}\left(q_{p}^{2 *}\right)\right), q_{p}^{2 *}\right) \\
& =r\left(d_{p u}^{\prime \prime}\left(q_{p}^{2 *}\right), q_{p}^{2 *}\right)+S_{u}\left(d_{p u}^{\prime \prime}\left(q_{p}^{2 *}\right)-d_{p u}^{\prime}\left(q_{p}^{2 *}\right)\right) .
\end{aligned}
$$

From (46), (106), (114)-(117) and $R>S_{o}+S_{u} \quad, \quad$ we know $u\left(r\left(d_{p l}^{\prime}\left(q_{p}^{2 *}\right), q_{p}^{2 *}\right)\right)<u\left(r\left(d_{p u}^{\prime}\left(q_{p}^{2 *}\right), q_{p}^{2 *}\right)\right)$, which conflicts with (112). As a result, $q_{p}^{1 *} \leq q_{p}^{2 *}$.

Lemma 23 shows that the increase of the uncertainty of the demand can make an active retailer order more and make a passive retailer order less but does not have any effect on the apprehensive and daring retailers.

\section{Numerical Example}

A fashion store, located in Yokohama, Japan, is planning to order a new design fashion sportswear. The unit wholesale price $W$, the unit revenue $R$, the unit salvage price $S_{o}$ and the unit opportunity cost $S_{u}$ are 7,10,1 and 4 (thousand JPY), respectively. The initial range of the possible demand is $[300,800]$. Let us consider how many goods the retailer should order.

Due to the new style there is no historical data available for statistical demand analysis. However, it is useful to consult some expert to make a judgment on the demand. Based on the knowledge of the expert, the initial reference range is divided into five subsections--- $[300,400)$, $[400,500),[500,600),[600,700)$ and $[700,800]$. The expert makes a pairwise comparison of the possibilities of different subsections and gives a pairwise comparison matrix as follows:

$$
A=\left[\begin{array}{ccccc}
1 & 1 / 2 & 1 / 5 & 1 / 3 & 1 \\
2 & 1 & 1 / 3 & 1 / 2 & 1 \\
5 & 3 & 1 & 1 & 4 \\
3 & 2 & 1 & 1 & 2 \\
1 & 1 & 1 / 4 & 1 / 2 & 1
\end{array}\right]
$$


The largest eigenvalue of the matrix $A$ is $\lambda_{\max }=5.07$, its normalized eigenvector is $\vec{p}=[0.22,0.35,1.00,0.73,0.29]^{t}$. The coordinates of the middle points of subsections from left to right is $350,450,550,650$ and 750 ; and their possibility degrees are set as $0.22,0.35,1.00$, 0.73 , and 0.29 , respectively. Supposing the possibility distribution of the demand is a triangular function and using (59) and (60) with $x_{c}=550$, we obtain

$$
\pi(x)=\left\{\begin{array}{l}
0, \quad x<294 \\
3.91 \times 10^{-3} x-1.15, \quad 294 \leq x \leq 550 \\
-2.70 \times 10^{-3} x+2.49, \quad 550 \leq x \leq 920 \\
0, \quad x>920
\end{array} .\right.
$$

The revised range of the possible demand is [294,920], that is, $d_{l}=294$ and $d_{u}=920$. By using (42), the profit function is

$$
r(x, q)=\left\{\begin{array}{ll}
9 x-6 q, & x<q \\
7 q-4 x, & x \geq q
\end{array} .\right.
$$

The highest profit is $r_{u}=(R-W) d_{u}=2760$ (thousand JPY) and the lowest profit is $r_{l}=d_{l} R+\left(d_{u}-d_{l}\right) S_{o}-d_{u} W=-2874$ (thousand JPY). The satisfaction function is set as

$$
\begin{aligned}
u(r(x, q)) & =\frac{r(x, q)-r_{l}}{r_{u}-r_{l}} \\
& =\left\{\begin{array}{ll}
1.6 \times 10^{-3} x-1.1 \times 10^{-3} q+5.1 \times 10^{-1}, & x<q \\
1.2 \times 10^{-3} q-0.7 \times 10^{-3} x+5.1 \times 10^{-1}, & x \geq q
\end{array},\right.
\end{aligned}
$$

where $294 \leq x, q \leq 920$. By using (61), (62), (75) and (80), we obtain $q_{1}^{*}=619, q_{2}^{*}=529$, $q_{3}^{*}=487$ and $q_{4}^{*}=920$. Clearly, we have $q_{3}^{*}<q_{2}^{*}<q_{1}^{*}<q_{4}^{*}$ which shows that the order quantity of the apprehensive retailer is less than the one of the passive retailer; the order quantity of the passive retailer is less than the one of the active retailer; the order quantity of the active retailer is less than the one of the daring retailer. Such results are quite in agreement with the situations encountered in the real business world.

\section{Conclusions}

This research analyzes the newsvendor problems for innovative products. Following the same idea of Fisher (1997), the innovative products are featured by the unpredictable demand and short life cycles. For characterizing the uncertainty of the demand, the possibility distribution is 
used and a simple and effective method for identifying the possibility distribution is proposed. The obtained possibility distribution reflects the expert's knowledge on the demand of the innovative product.

Due to the shorter life cycle than the procurement lead-time, determining the order quantity is a typical one-shot decision problem. Instead of using the subjective expected utility theory (SEU), we utilize the one-shot decision theory (OSDT) to analyze the newsvendor problems. The proposed models are scenario-based decision models; they are fundamentally different from the newsvendor models with SEU which are lottery-based models.

In this paper, the general solutions of active, passive, apprehensive and daring focus points and optimal alternatives are proposed and the existence theorem is established in the one-shot decision theory.

Newsvendor models with four types of focus points are developed for four types of retailers; i.e., the active retailer, the passive retailer, the apprehensive retailer and the daring retailer. The active retailer takes into account a demand with a higher satisfaction and a higher possibility; the passive retailer focuses on a demand with a lower satisfaction and a higher possibility; the apprehensive retailer thinks over a demand with a lower satisfaction and a lower possibility; the daring retailer considers a demand with a higher satisfaction and a lower possibility. The optimal order quantities for these four types of retailers are obtained and we have the following conclusions:

(1) The focus point of the active retailer's optimal order quantity is the optimal order quantity itself. It means that the active retailer has confidence that he/she can sell all the products that he/she has optimally ordered.

(2) The passive retailer chooses the optimal order quantity which makes its two focus points have the same possibility degrees and the same satisfaction levels.

(3) The apprehensive retailer takes into account two extreme demands (the highest and the lowest demand) and chooses the optimal order quantity which makes the satisfaction levels of the highest demand and the lowest demand equal.

(4) For the daring retailer, the highest demand is his/her optimal order quantity and he/she believes all ordered products can be sold.

(5) The optimal daring order quantity is always larger than any other type of optimal order quantity. If the possibility distribution is symmetric, the optimal active order quantity is larger than the optimal passive one; the optimal passive order quantity is larger than the optimal apprehensive one.

(6) Setting the satisfaction function as a linear function, the optimal active order quantity and its focus point are decreasing in the unit wholesale price, increasing in the unit revenue and the unit salvage price. The unit opportunity cost has no effect on them. 
(7) The passive retailer offsets the loss caused by the increase of the unit opportunity cost by increasing the order quantity.

(8) The optimal apprehensive order quantity increases in the unit salvage price and the unit opportunity cost, decreases in the unit revenue. The unit wholesale price has no effect on it.

(9) The increase of the uncertainty of the demand can make an active retailer order more and make a passive retailer order less but does not have any effect on the apprehensive and daring retailers.

The above results provide managerial insights into the behaviors of different types of retailers. The research on newsvendor problems for innovative products with OSDT is at an early stage. This research provides a theoretical base for the further researches. As a direct extension of this study, supply chain coordination problems for innovative products will be studied.

\section{References}

Brito, A.J., de Almeida, A.T., 2012. Modeling a multi-attribute utility newsvendor with partial backlogging. European Journal of Operational Research 220 (3) 820-830.

Caliskan-Demirag, O, Chen, Y., Li, J., 2011. Customer and retailer rebates under risk aversion. International Journal of Production Economics 133 (2) 736-750.

Chen, J., 2011. Returns with wholesale-price-discount contract in a newsvendor problem. International Journal of Production Economics 130 (1) 104-111.

Chen, S.P., Ho, Y.H., 2011. Analysis of the newsboy problem with fuzzy demands and incremental discounts. International Journal of Production Economics 129 (1)169-177.

Dutta, P., Chakraborty, D., 2010. Incorporating one-way substitution policy into the newsboy problem with imprecise customer demand. European Journal of Operational Research 200 (1) 99-110.

Fisher, M.L., 1997. What is the right supply chain for your product? Harvard Business Review (March-April) 105-116.

Grubbstrom, R.W., 2010. The newsboy problem when customer demand is a compound renewal process. European Journal of Operational Research 203 (1) 134-142.

Guo, P., Tanaka, H., 2003. Decision analysis based on fused double exponential possibility distributions. European Journal of Operational Research 148 (3) 467-479.

Guo, P., Tanaka, H., 2010. Decision making with interval probabilities. European Journal of Operational Research 203 (2) 444-454.

Guo, P., 2010a. One-shot decision approach and its application to duopoly market. International Journal of Information and Decision Sciences 2 (3) 213-232.

Guo, P., 2010b. Private real estate investment analysis within a one-shot decision framework. International Real Estate Review 13 (3) 238-260. 
Guo, P., Yan, R., Wang, J., 2010. Duopoly market analysis within one-shot decision framework with asymmetric possibilistic information. International Journal of Computational Intelligence System 3 (6) 786-796.

Guo, P., 2011. One-shot decision theory. IEEE Transactions on SMC, Part A: Systems and Humans 41 (5) 917-926.

Guo, P., Wang, Y., 2012. Eliciting dual interval probabilities from interval comparison matrices. Information Sciences 190 (1) 17-26.

Guo, P., 2014. One-shot decision theory: A fundamental alternative for decision under uncertainty. In P. Guo and W. Pedrycz (Eds.), Human-Centric Decision-Making Models for Social Sciences. Studies in Computational Intelligence (Vol. 502, pp. 33-55). Berlin Heidelberg: Springer-Verlag.

Guo, P., Y. Li, 2014. Approaches to multistage one-shot decision making. European Journal of Operational Research. http://dx.doi.org/10.1016/j.ejor.2013.12.038.

Ishii, H., Konno, T., 1998. A stochastic inventory problem with fuzzy shortage cost. European Journal of Operational Research 106 (1) 90-94.

Kao, C., Hsu., W.K., 2002. A single-period inventory model with fuzzy demand. Computers and Mathematics with Applications 43 (6-7) 841-848.

Khouja, M., 1999. The single-period (news-vendor) problem: Literature review and suggestions for future research. Omega 27 (5) 537-553.

Kwon, K., Cheong, T., 2014. A minimax distribution-free procedure for a newsvendor problem with free shipping. European Journal of Operational Research 232 (1) 234-240.

Li, L., Kabadi, S. N., Nair, K.P.K., 2002. Fuzzy models for single-period inventory problem. Fuzzy Sets and Systems 132 (3) 273-289.

Madden, P., 1986. Concavity and optimization in microeconomics. Oxford: Blackwell.

Murray, C.C., Gosavi, A., Talukdar, D., 2012. The multi-product price-setting newsvendor with resource capacity constraints. International Journal of Production Economics 138 (1) 148-158.

Petrovic, D., Petrovic, R., Vujosevic, M., 1996. Fuzzy models for the newsboy problem. International Journal of Production Economics 45 (1-3) 435-441.

Petruzzi, N.C., Dada, M., 1999. Pricing and the newsvendor problem: A review with extensions. Operations Research 47 (2) 183-194.

Qin, Y., Wang, R., Vakharia, A.J., Chen, Y., Seref, M.M.H., 2011. The newsvendor problem: Review and directions for future research. European Journal of Operational Research 213 (2) 361-374.

Ryu, K., Yucesan, E., 2010. A fuzzy newsvendor approach to supply chain coordination. European Journal of Operational Research 200 (2) 421-438. 
Saaty, T.L., 1980. The Analytic Hierarchy Process. NY: McGraw Hill.

Salinger, M., Ampudia, M., 2011. Simple economics of the price-setting newsvendor problem. Management Science 57 (11) 1996-1998.

Seifert, R.W., Zequeira, R.I., Liao, S., 2012. A three-echelon supply chain with price-only contracts and sub-supply chain coordination. International Journal of Production Economics 138 (2) 345-353.

Sion, M., 1958. On general minimax theorems. Pacific Journal of Mathematics 8 (1) 171-176.

Summerfield, N.S., Dror, M., 2012. Stochastic programming for decentralized newsvendor with transshipment. International Journal of Production Economics 137 (2) 292-303.

Wang, C.X., Webster, S., Suresh, N.C., 2009. Would a risk-averse newsvendor order less at a higher selling price? European Journal of Operation Research 196 (2) 544-553.

Wang, C.X., Webster, S., 2009. The loss-averse newsvendor problem. Omega 37(1) 93-105.

Wang, C.X., 2010. The loss-averse newsvendor game. International Journal of Production Economics 124 (2) 448-452.

Wang, J.C., Lau, A.H.L., Lau, H.S., 2012. Practical and effective contracts for the dominant retailer of a newsvendor product with price-sensitive demand. International Journal of Production Economics 138 (1) 46-54.

Wu, Z., Crama, P., Zhu, W., 2012. The newsvendor's optimal incentive contracts for multiple advertisers. European Journal of Operational Research 220 (1) 171-181.

Xu, R., Zhai, X., 2008. Optimal models for single-period supply chain problems with fuzzy demand. Information Sciences 178 (17) 3374-3381.

Xu, X., Cai, X., Chen, Y., 2011. Unimodality of price-setting newsvendor's objective function with multiplicative demand and its applications. International Journal of Production Economics 133 (2) 653-661. 